\title{
R\&D for a Soft X-Ray Free Electron Laser Facility
}

\section{A White Paper}

Report prepared by LBNL and SLAC with contributions from

LBNL: David Attwood, John Byrd, John Corlett, Peter Denes, Roger Falcone, Phil Heimann, Wim Leemans, Howard Padmore, Soren Prestemon, Fernando Sannibale, Ross Schlueter, Carl Schroeder, John Staples, Marco Venturini, Tony Warwick, Russell Wells, Russell Wilcox, and Alexander Zholents

SLAC: Chris Adolphsen, John Arthur, Uwe Bergmann, Yunhai Cai, Eric Colby, David Dowell, Paul Emma, John Fox, Josef Frisch, John Galayda, Robert Hettel, Zhirong Huang, Nan Phinney, Tom Rabedeau, Tor Raubenheimer, David Reis, John Schmerge, Joachim Stöhr, Gennady Stupakov, Bill White, and Dao Xiang

\section{Lawrence Berkeley National Laboratory SLAC National Accelerator Laboratory}

June 2009 
Lawrence Berkeley National Laboratory

University of California

Berkeley, CA 94720

SLAC National Accelerator Laboratory

2575 Sand Hill Road

Menlo Park, CA 94025

\section{Editor:}

Arthur L. Robinson (LBNL)

\section{DISCLAIMER}

This document was prepared as an account of work sponsored by the United States Government. While this document is believed to contain correct information, neither the United States Government nor any agency thereof, nor any of their employees, nor any of their contractors, subcontractors, or their employees, makes any warranty, express or implied, or assumes any legal liability or responsibility for the accuracy, completeness, or any third party's use or the results of such use of any information, apparatus, product, or process disclosed, or represents that its use would not infringe privately owned rights. Reference herein to any specific commercial product, process, or service by its trade name, trademark, manufacturer, or otherwise, does not necessarily constitute or imply its endorsement, recommendation, or favoring by the United States Government or any agency thereof or its contractors or subcontractors. The views and opinions of authors expressed herein do not necessarily state or reflect those of the United States Government or any agency thereof or its contractors or subcontractors.

This work was supported by the Director, Office of Science, Office of Basic Energy Sciences, of the U.S. Department of Energy under Contracts No. DE-AC02-05CH11231 (LBNL) and DE-AC02-76SF00515 (SLAC). 


\section{Table of Contents}

EXECUTIVE SUMMARY

1 INTRODUCTION

$\begin{array}{lll}1.1 & \text { Future Science Drivers } & 7\end{array}$

$\begin{array}{llr}1.2 & \text { Soft X-Ray FEL Light Source Capabilities } & 8\end{array}$

$\begin{array}{lll}1.3 & \text { Linac-Based Free Electron Lasers } & 9\end{array}$

1.4 R\&D for FEL Accelerator Systems and Components $\quad 11$

1.5 Major Accelerator Parameters Needed to Meet Science Drivers 12

2 SOFT X-RAY FEL TECHNOLOGY R\&D 15

$\begin{array}{llr}2.1 & \text { Injectors } & 15\end{array}$

2.1.1 Electron Gun 15

$\begin{array}{ll}\text { 2.1.2 Cathode/laser-system choice } & 17\end{array}$

2.1.3 Proposed injector R\&D activity 19

$\begin{array}{llr}2.2 & \text { FEL Design } & 22\end{array}$

$\begin{array}{llr}2.3 & \text { Insertion Devices } & 27\end{array}$

2.4 Linac Systems $\quad 29$

2.4.1 Linac parameters 29

$\begin{array}{ll}2.4 .2 & \text { Cavity design }\end{array}$

2.4.3 Cryogenic load 31

2.4.4 Cryomodules 31

2.4.5 LLRF and HLRF $\quad 32$

2.4.6 Pre-Construction R\&D Plan 32

2.5 Laser Systems

2.5.1 Injector drive lasers $\quad 33$

2.5.2 Beam manipulation and seeding 33

$\begin{array}{lll}2.5 .3 & \text { Synchronization } & 34\end{array}$

3 COST ESTIMATE $\quad 35$

4 CORE COMPETENCIES OF INSTITUTIONS 36

4.1 LBNL $\quad 36$

4.1.1 Research facilities $\quad 36$

$\begin{array}{lll}\text { 4.1.2 R\&D support } & 37\end{array}$

$\begin{array}{llr}\text { 4.2 } & \text { SLAC } & 38\end{array}$

4.2.1 Research facilities $\quad 39$ 
5 OTHER INSTITUTIONS $\quad 42$

6 REFERENCES $\quad 43$

7 ACKNOWLEDGEMENTS $\quad 46$

8 APPENDIX. RESUMES OF PRINCIPAL PARTICIPANTS 47 


\section{Executive Summary}

Several recent reports have identified the scientific requirements for a future soft x-ray light source, and a high-repetition-rate free-electron laser (FEL) facility that is responsive to these requirements is now on the horizon. $\mathrm{R} \& \mathrm{D}$ in some critical areas is needed, however, to demonstrate technical performance, thus reducing technical risks and construction costs. Such a facility most likely will be based on a CW superconducting linear accelerator with beam supplied by a high-brightness, high-repetition-rate photocathode electron gun operating in CW mode, and on an array of FELs to which the accelerated beam is distributed, each operating at high repetition rate and with even pulse spacing. Dependent on experimental requirements, the individual FELs can be configured for either self-amplified spontaneous emission (SASE), seeded, or oscillator mode of operation, including the use of high-gain harmonic generation (HGHG), echo-enhanced harmonic generation (EEHG), harmonic cascade, or other configurations. In this White Paper we identify the overall accelerator R\&D needs, and highlight the most important pre-construction $R \& D$ tasks required to value-engineer the design configuration and deliverables for such a facility. In Section 1.4 we identify the comprehensive R\&D ultimately needed. We identify below the highest-priority requirements for understanding machine performance and reduce risk and costs at this pre-conceptual design stage. Details of implementing the required tasks will be the subject of future evaluation.

- Our highest-priority R\&D program is the injector, which must be capable of delivering a beam with bunches up to a nanocoulomb at $\mathrm{MHz}$ repetition rate and with normalized emittance $\leq 1 \mathrm{~mm} \bullet \mathrm{mrad}$. This will require integrated accelerating structure, cathode, and laser systems development. Cathode materials will impact the choice of laser technology in wavelength and energy per pulse, as well as vacuum requirements in the accelerating structure.

- Demonstration experiments in advanced seeding techniques, such as EEHG, and other optical manipulations to enhance the FEL process are required to reduce technical risk in producing temporally coherent and ultrashort x-ray output using optical seed lasers. Success of EEHG in particular would result in reduced development and cost of laser systems and accelerator hardware for seeded FELs.

- With a 1.5-2.5 GeV linac, FELs could operate in the VUV-soft x-ray range, where the actual beam energy will be determined by undulator technology; for example, to use the lower energy would require the use of advanced designs for which undulator R\&D is needed. Significant reductions in both unit costs and accelerator costs resulting from the lower electron beam energy required to achieve lasing at a particular wavelength could be obtained with undulator development. Characterization of the wakefields of the vacuum chambers in narrow-gap undulators will be needed to minimize risk in ability to deliver close to transform limited pulses.

- CW superconducting RF technology for an FEL facility with short bunches at MHz rate and up to $\mathrm{mA}$ average current will require selection of design choices in cavity frequency and geometry, higher order mode suppression and power dissipation, RF power supply and distribution, accelerating gradient, and cryogenics systems. $R \& D$ is needed to define a cost and performance optimum. 
- Developments in laser technology are proceeding at rapid pace, and progress in high-power lasers, harmonic generation, and tunable sources will need to be tracked. 


\section{Introduction}

Several recent reports provide a summary of the scientific challenges of the future and needs for $\mathrm{x}$-ray tools to address them $[1,2,3,4,5]$. Here, we summarize the scientific goals and the essential x-ray capabilities for a future soft x-ray free-electron laser (FEL) facility, and the critical R\&D required to realize such capabilities.

\subsection{Future Science Drivers}

The innovation expected from the construction and operation of enhanced x-ray sources lies in combining and extending existing knowledge of the nanoworld, with extreme spatial, temporal, and energy resolution. Properties of anticipated new x-ray sources include the ability to reach to the frontier of ultrafast timescales of electron motion around an atom, the spatial scale of the atomic bond, and the energy scale of the bond that holds electrons in correlated motion with near neighbors. FEL x-ray sources offer unique capabilities to combine information on atomic length scales with data on full-scale functional systems under realistic operational conditions. In addition, these novel sources have an intensity and brightness needed to observe the subtlest of nature's secrets at these frontier space, time, and energy scales.

While our understanding of the time- and energy-dependent behavior of matter in its ground or natural state is still severely limited, we know even less about excited states. Such states are of key scientific and technological importance, for they typically determine how matter functions during chemical reactions and during physical and biological processes. Excited states of interest span a vast range, being close to the natural (ground or equilibrium, as appropriate) state (as in electronic transport), relatively far from it (as in photo-chemical reactions), and very far from it (as in extreme conditions that can be imposed by pressure, radiation, or electric and magnetic fields).

Embedded in these challenges is the exploration of the atomic or nanoscale on the "natural" time scale of atoms, electrons, and spins and on the "operational" time scale that determines function and is the key in technological applications. There is presently a striking discrepancy between the natural time scales of atomic motion (about $100 \mathrm{fs}$ ), spin motion (down to about $1 \mathrm{fs}$ ), and electronic motion (down to attoseconds) and the fastest operational timescales (approaching $100 \mathrm{ps}$ ). A critical scientific challenge is to pave the way for technology to expand into the ultrafast, with the opportunity of five orders of magnitude improvement down to the intrinsic time scale of charge and spin motions of valence electrons.

In addition to temporal resolution, spatial resolution at the length scales associated with functional systems is also important on both short and longer time scales characteristic of diffusion and displacement, and of sound propagation, for example. Higher coherent flux from future x-ray sources will permit imaging on time scales approaching MHz. This opens the door to scientific discovery in catalysis, deformation, phase nucleation and transformations.

The unique strength of future x-ray sources lies in their ability to combine coverage of the atomic and nanoworld with the entire time domain from seconds to attoseconds and a spatial domain from a tenth of a nanometer to a millimeter. 


\subsection{Soft X-Ray FEL Light Source Capabilities}

Here we briefly describe key performance areas where significant advances in x-ray capabilities are both required by the science, and technically feasible in the near-term. Not only are these individual requirements beyond the capabilities of present light sources, the paramount scientific challenges often require a source providing several of these features simultaneously.

- X-ray time structure-complete control of longitudinal phase space

- Pulse duration and temporal resolution extending to the attosecond regime

- Fourier-transform-limited (FTL) pulse structure extending from few-picosecond pulses with meV bandwidths to sub-femtosecond pulses with $10-\mathrm{eV}$ bandwidths

- Control of longitudinal pulse shape, amplitude and phase

○ Synchronization and full integration with conventional pulsed laser sources.

- Full transverse coherence

$\circ$ Requirements set by real-space imaging, diffractive imaging, and photoncorrelation spectroscopy

O Optical systems to preserve and exploit transverse coherence.

- High peak flux and brightness

$\circ$ Requirements set by non-linear effects in materials.

- High average flux and brightness

- CW sources with average flux and/or brightness substantially beyond existing sources in the soft $x$-ray range

- Short-pulse sources with high repetition rates $(100 \mathrm{kHz})$ providing average flux and/or brightness substantially beyond existing sources in the hard and/or soft $\mathrm{x}$-ray range.

- Tunability, polarization control, and extended photon energies

○ Soft x-ray tunability (throughout the transition-metal L-edges) and polarization control and modulation.

The FEL facilities in operation or under construction, including the first hard x-ray FEL, the LCLS, will lead to new science and open up research not currently possible. By utilizing more advanced technologies of high-repetition-rate (of order MHz), low-emittance electron injectors, $\mathrm{CW}$ superconducting RF (SCRF) linac technology, and optical manipulation techniques, the next generation of FEL facility will be able to open up additional areas of research, complementing the FEL facilities currently operational or being built, as well as the existing third-generation light sources. In Table 1 the performance of this type of machine is indicated, where the flexible seeded-FEL approach provides for three distinct capabilities in a single facility:

- Controlled pulses of 10-100 fs duration for ultrafast experiments in atomic and molecular dynamics

- Sub-femtosecond capability with pulses of $\sim 100$ as duration for ultrafast experiments in electronic dynamics 
- Temporally coherent pulses of 100-1000 fs duration for experiments in ultrahigh resolution spectroscopy and imaging

Table 1.1. Performance goals of three major beamline types for a future soft x-ray FEL facility

\begin{tabular}{|c|c|c|c|}
\hline & $\begin{array}{c}\text { Ultrashort pulse } \\
\text { beamlines }\end{array}$ & $\begin{array}{l}\text { Short pulse } \\
\text { beamlines }\end{array}$ & $\begin{array}{l}\text { High spectral } \\
\text { resolution beamlines }\end{array}$ \\
\hline Pulse length (fs) & $0.1-10$ & $10-100$ & $100-1000$ \\
\hline Wavelength range $(\mathrm{nm})$ & $1-30$ & $1-100$ & $1-100$ \\
\hline Repetition rate $(\mathrm{kHz})$ & $1-10$ (goal 100) & 100 & 100 \\
\hline Peak power (GW) & $0.1-1$ & $1-5$ & $1-5$ \\
\hline Photons/pulse (@1 nm) & $2 \times 10^{8}$ (in 100 as) & $2 \times 10^{11}$ (in $100 \mathrm{fs}$ ) & $2 \times 10^{12}$ (in $\left.500 \mathrm{fs}\right)$ \\
\hline Bandwidth & $\begin{array}{l}\text { Fourier transform limit } \\
\qquad(\mathrm{FTL})\end{array}$ & few $\times$ FTL & few $x$ FTL \\
\hline $\begin{array}{l}\text { Photons/pulse (@ 3rd } \\
\text { harmonic } 0.3 \mathrm{~nm} \text { ) }\end{array}$ & $2 \times 10^{6}$ (in 100 as) & $2 \times 10^{9}$ (in $100 \mathrm{fs}$ ) & $2 \times 10^{10}$ (in 500 fs) \\
\hline Polarization & Variable, linear/circular & Variable, linear/circular & Variable, linear/circular \\
\hline $\begin{array}{l}\text { Peak brightness } \\
\left.\text { (ph/s/mm } / \mathrm{mr}^{2} / 0.1 \% \mathrm{BW}\right)\end{array}$ & $\sim 10^{26}$ & $\sim 10^{29}$ & $\sim 10^{30}$ \\
\hline $\begin{array}{l}\text { Average brightness } \\
\left.\text { (ph/s/mm } / \mathrm{mr}^{2} / 0.1 \% \mathrm{BW}\right)\end{array}$ & $\sim 10^{21}$ & $\sim 10^{24}$ & $\sim 10^{25}$ \\
\hline
\end{tabular}

\subsection{Linac-Based Free Electron Lasers}

A free-electron laser is a source of intense coherent radiation that may be produced when an electron beam of suitable beam quality and energy is propagated through an undulator. The radiation process in an FEL introduces periodic enhancement of the charge density in the electron bunch, forming micro-bunches separated by a distance equal to the wavelength of the emitted radiation. These micro-bunches begin to radiate coherently, significantly increasing the peak brightness. FELs can be either of two general types: (1) a single pass, high-gain type or (2) an oscillator type making use of high reflectivity mirrors. The single pass, high-gain type can further be operated in two modes, SASE (self-amplified spontaneous emission) and seeded. In SASE FELs, the microbunching arises from the interaction of the electron beam and the synchrotron radiation emitted upstream in the undulator; seeded FELs introduce an external coherent light pulse that produces microbunching in the electron beam. In an oscillator FEL, a light field develops inside an optical cavity as the undulator radiation from previous passes is trapped and filtered by the mirrors and amplified by interacting with new electron bunches in the undulator. The peak spectral brightness of an FEL can be billions of times higher than that of a typical insertion device in a storage ring. In addition, light from an FEL has full transverse coherence and, in some FELs, a very high degree of a temporal coherence. Using the same electron beam in FELs and other coherent synchrotron radiation devices, each at a different wavelength (such as an undulator downstream of the FEL tuned to a longer wavelength), offers 
capabilities for synchronized multi-color sources. Future FEL performance may encompass a wide range of x-ray parameters, dependent on details of design.

The electron beam quality for an x-ray FEL must exceed that of a storage ring, and this is typically provided by a linac. The electron beam itself must have laser-like quality, with transverse geometric emittances $\left(\varepsilon_{g \perp}\right)$ comparable to the diffraction limit of the emitted light $\varepsilon_{g \perp} \approx \lambda_{x-\text { ray }} / 4 \pi$, small uncorrelated energy spread of less than a few hundred $\mathrm{keV}$, and peak current of hundreds of amperes to kiloamperes. Geometric emittance and normalized emittance $\varepsilon_{n \perp}$ are related by $\varepsilon_{g \perp}=\varepsilon_{n \perp} / \gamma$, where $\gamma$ is the relativistic factor. The undulator resonance condition determines the electron energy to obtain short wavelength radiation: $\lambda_{x-\text { ray }}=\lambda_{u}\left(1+K^{2} / 2\right) / 2 \gamma^{2}, \lambda_{u}$ is the undulator period, and $K=e B \lambda_{u} / 2 \pi m c^{2}>1$ is the undulator parameter. Another way to describe beam quality is the electron beam brightness: $\widehat{B}=2 I / \varepsilon_{n \perp}^{2}$, where $I$ is the peak electron current. In fact, brightness largely defines the FEL gain length, i.e., the number of the undulator periods $N_{g}$ that the electron bunch passes while the x-ray radiation gains a factor of $\mathrm{e}=2.718$ in power: $N_{g}=(4 \pi \sqrt{3} \rho)^{-1}$, where $\rho$ is a so-called Pierce parameter. In a $1 \mathrm{D}$ approximation $\rho \propto \widehat{B}^{1 / 3}$.

Existing FELs operate in SASE and will provide short x-ray pulses (tens to hundreds of femtosecnds), of unprecedented peak power (up to ten of gigawatts), and peak brightness is expected to be of the order of $10^{33}$ photons $/ \mathrm{s} / \mathrm{mm}^{2} / \mathrm{mrad}^{2}$ per $0.1 \%$ bandwidth, many orders of magnitude greater than storage ring or ERL (energy recovery linac) incoherent sources could provide. Implementation of optical manipulations could allow exquisite control of the electron beam, and development of techniques such as high-gain harmonic generation (HGHG), harmonic cascade, enhanced SASE (ESASE), echo-enhanced harmonic generation (EEHG), and selfseeding, are expected to enhance performance of planned and future FELs, by controlling x-ray pulse duration $(<1-100 \mathrm{~s}$ fs), imparting a very high degree of temporal coherence and thus $\mathrm{meV}$ resolving power, providing synchronization to another laser for pump-probe experiments, and increasing peak current, thus reducing the length of the radiator undulator required. Seeding by conventional lasers and harmonic generation in an FEL have been demonstrated at VUV wavelengths. Feedback control of the seed laser could shape the amplitude and phase, optimizing $\mathrm{x}$-ray pulse parameters for experiments.

Practical repetition rates for x-ray FELs are currently limited by high-brightness electron-gun technology, and the facilities currently under construction are planned to use macropulses at $\sim 10-120 \mathrm{~Hz}$ repetition rate. Pulsed linac repetition rates might eventually approach the order of $1 \mathrm{kHz}$ with developments in pulsed high power RF sources and accelerating structures. In bunchtrain mode, pulsed linacs can provide high bunch repetition rates (in principle up to the RF frequency of the linac) over the duration of the linac RF pulse (approaching milliseconds for superconducting linacs) to achieve average bunch repetition rates that may reach the order of $10^{5}$ bunches/second. In contrast to the high bunch rates achieved in bursts with pulsed linacs, $\mathrm{CW}$ superconducting-linac-based FELs promise to provide high pulse repetition rate with even spacing, from tens of $\mathrm{kHz}$ to tens of $\mathrm{MHz}$ or greater as electron gun technology is developed. CW superconducting-linac-based FELs offer a route to higher average brightness with a timestructured beam that is well suited to many experimental needs, particularly pump-probe 
experiments. The bunches from the linac can be fanned out to multiple undulators in a fully programmable fashion. CW operation of the accelerator, with feedback loops to control beam position and energy, offers improved stability over pulsed machines. Higher repetition rates could be supported by the linac, and CW linacs offer the potential for increased average brightness as technology is developed.

\subsection{R\&D for FEL Accelerator Systems and Components}

The next-generation of light sources will need extensive R\&D to define the final optimum configuration. This is in contrast to the evolution of storage-ring-based synchrotron-radiation facilities, which were highly refined elaborations based upon well-understood concepts and technologies not far removed from their origins in high-energy physics, and design confidence could largely be achieved from experience augmented by simulation. This White Paper highlights pre-construction R\&D that addresses the most pressing needs of a future highrepetition-rate soft x-ray FEL light source. Our focus is on the early R\&D needed to reduce technical risk and allow definition of the machine configuration and performance deliverables, and reliably understand costs. An ultimate experimental user facility will bring additional R\&D challenges, which would naturally be addressed as part of a construction project. Similarly, several key R\&D elements will transform in nature from early conceptual R\&D into more detailed engineering design and optimization as part of a construction project. Below, we list the areas where R\&D is ultimately needed, and in the following section we prioritize and select the most critical pre-construction R\&D needs, those that will have the greatest impact. What is ultimately needed is a comprehensive $R \& D$ program that develops advanced technologies for:

- High-repetition-rate, low-emittance electron injectors

- High quantum efficiency photocathodes

○ High-gradient accelerating structures

- High-power drive laser that matches the photocathode materials properties and that has pulse shaping $(\mathrm{x}, \mathrm{y}, \mathrm{t})$.

- FEL design

- Emittance control and manipulation techniques

- Optimal bunch compression schemes

$\circ$ High repetition-rate beam switching

- High-brightness beam transport

○ High resolution start-to-end modeling

- Optical manipulations of beams

- To control pulse duration

- To impart temporal coherence

- For control of SASE

- For seeding.

- CW superconducting RF accelerating structures

○ Cavity design

○ High-gradient 
○ High-Q

- Parasitic mode power handling

- RF power distribution.

- High-efficiency $2 \mathrm{~K}$ cryogenics systems.

- High-power seed laser and pump laser systems

- Repetition rate from $10 \mathrm{kHz}$ to $\mathrm{MHz}$

- Harmonic generation

○ Tunability

- Filtering (spatial and temporal).

- Harmonic cavities.

- Deflecting cavities.

- Short-period undulator design

- Superconducting technologies to eliminate moving parts and reduce costs

- Elliptical polarization capability.

- Timing \& synchronization systems

○ Sub-femtosecond stability.

- High-resolution electron beam diagnostics.

- Radiation protection and active interlock systems.

In addition to these $R \& D$ needs for the source, significant $R \& D$ will be needed on the experimental side, for example on

- X-ray optics, characterization, and manipulations

○ Robust mirror materials and optical coatings

- Ultrashort pulse and high resolution beamline design

- Integration of beamline design into FEL and facility layout

- Modeling of coherence with real optical surfaces

- Experimental work on nonlinear optics and laser-X-ray cross correlation.

- Detectors able to realize the benefits of such a versatile source.

\subsection{Major Accelerator Parameters Needed to Meet Science Drivers}

A high-repetition-rate FEL facility that is responsive to the technical requirements described in Section 1.2 is on the horizon. R\&D in some critical areas is needed, however, to demonstrate technical performance and to reduce costs. Such a facility could be based on a CW superconducting linear accelerator with beam supplied by a high-brightness, high-repetition-rate photocathode electron gun operating in CW mode, on an array of FELs to which the accelerated beam is distributed, and on a set of FEL beamlines operating at high repetition rate and with even pulse spacing. Dependent on experimental requirements, the individual FELs can be configured 
for either SASE, seeded, or oscillator mode of operation, including use of HGHG, EEHG, harmonic cascade, or other configuration. Such an array of FELs could obtain the x-ray pulse parameters in Table 1.1. Figure 1.1 shows a schematic of the type of machine configuration that could be built.

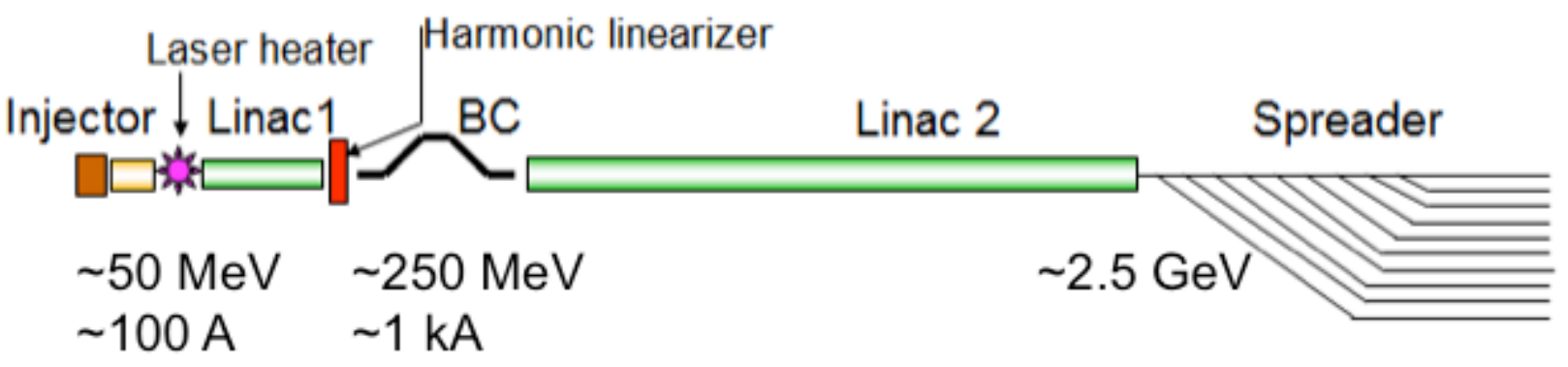

Figure 1.1. Schematic of a high repetition-rate accelerator for a FEL facility that is responsive to the technical requirements described in Section 1.2. Major machine elements are identified, BC is a bunch compressor, beam energies and peak currents are illustrative.

With a $1.5-2.5 \mathrm{GeV}$ linac, FELs could operate in the VUV-to-soft x-ray range, where the actual beam energy will be determined by undulator technology; for example, to use the lower energy would require the use of advanced designs for which undulator R\&D is needed. To achieve the performance parameters of Table 1 requires a source delivering beams with bunches up to a nanocoulomb at a uniform $\mathrm{MHz}$ repetition rate and with normalized emittance $\leq 1 \mathrm{~mm} \bullet \mathrm{mrad}$. Bunches at the FELs may be from 100s of femtoseconds long to as short as tens of femtoseconds and perhaps even shorter, and with charge from 1 nanocoulomb for long bunches to tens of picocoulombs for short bunches, while maintaining a peak current of approximately $1 \mathrm{kA}$. Uncorrelated energy spread should be less than a few hundred $\mathrm{keV}$, and normalized slice emittance $\leq 1 \mathrm{~mm} \bullet \mathrm{mrad}$. CW superconducting RF technology will be required to accommodate the $\mathrm{MHz}$ bunch rate, and a beam switchyard with deflecting elements operating at up to $100 \mathrm{kHz}$ repetition rate (adjustable) and shot-to-shot amplitude stability better than $10^{-3}$ to distribute bunches to an array of up to ten FELs. Optical manipulation techniques will be required to seed temporally coherent $\mathrm{x}$-ray output, and to control SASE in some FEL configurations.

Table 1.2 summarizes the accelerator parameters that require pre-construction $\mathrm{R} \& \mathrm{D}$. We identify in Section 2 those areas in which $R \& D$ is required to demonstrate performance not yet achieved, and to support risk reductions in major cost drivers for a future soft x-ray FEL facility with performance outlined in Section 1.2. The goals of this R\&D are to support definition of the machine configuration and performance deliverables, as well as a reasonable cost estimate for a future construction project; it is not to engineer solutions for a specific design. 
Table 1.2. Major machine parameters, processes, or components driving pre-conceptual R\&D needs.

\begin{tabular}{|c|c|c|c|}
\hline $\begin{array}{l}\text { Parameter I } \\
\text { Process I } \\
\text { Component }\end{array}$ & Design goal & Notes & Impact \\
\hline $\begin{array}{l}\text { Electron beam } \\
\text { energy }\end{array}$ & $1.5-2.5 \mathrm{GeV}$ & $\begin{array}{l}\text { To lase at soft x-ray } \\
\text { wavelengths, dependent on } \\
\text { undulator resonance and } \\
\text { electron beam geometric } \\
\text { emittance }\end{array}$ & $\begin{array}{l}\text { Reduced costs from lower-energy } \\
\text { accelerator achieved with shorter- } \\
\text { period undulators and smaller } \\
\text { normalized emittance }\end{array}$ \\
\hline $\begin{array}{l}\text { Electron bunch rate } \\
\text { in linac }\end{array}$ & $1 \mathrm{MHz}$ & $\begin{array}{l}\text { High rep-rate for multiplexed } \\
\text { beams, and high average power } \\
\text { for each FEL } \\
\text { Requires high rep-rate electron } \\
\text { source }\end{array}$ & $\begin{array}{l}\text { Flexible facility offering wide range of } \\
\text { performance with individually } \\
\text { configured and controlled FELs } \\
\text { Serves a large number of users } \\
\text { Requires CW superconducting RF } \\
\text { systems }\end{array}$ \\
\hline Number of FELs & 10 & $\begin{array}{l}\text { Beam switchyard distributes } \\
\text { bunches from the linac to each } \\
\text { FEL } \\
\text { Beam switchyard has pulsed } \\
\text { elements to flexibly distribute } \\
\text { bunches at variable rate to each } \\
\text { FEL }\end{array}$ & $\begin{array}{l}\text { Flexible facility offering wide range of } \\
\text { performance with individually } \\
\text { configured and controlled FELs } \\
\text { Serves a large number of users }\end{array}$ \\
\hline $\begin{array}{l}\text { Electron bunch rate } \\
\text { per FEL }\end{array}$ & $\begin{array}{l}100 \mathrm{kHz} \text { (Potentially } \\
\text { up to } \mathrm{MHz} \text { for special } \\
\text { operations with single } \\
\text { beamline) }\end{array}$ & $\begin{array}{l}\text { Stripline kicker with high-voltage } \\
\text { pulsed power supply }\end{array}$ & High data acquisition rate, good $\mathrm{S} / \mathrm{N}$ \\
\hline $\begin{array}{l}\text { Beam switchyard } \\
\text { pulsed element } \\
\text { stability }\end{array}$ & Better than $10^{-3}$ & $\begin{array}{l}\text { Stripline kicker with high-voltage } \\
\text { pulsed power supply }\end{array}$ & $\begin{array}{l}\text { Reliable operation with stable FEL } \\
\text { output }\end{array}$ \\
\hline $\begin{array}{l}\text { Electron bunch } \\
\text { charge }\end{array}$ & $\sim 10 \mathrm{~s} \mathrm{pC}-1 \mathrm{nC}$ & $\begin{array}{l}\text { Dependent on FEL and } \\
\text { optimized for experimental } \\
\text { needs } \\
\text { Bunch current maintained } \sim 1 \mathrm{kA}\end{array}$ & $\begin{array}{l}\text { Flexibility in achieving design goals } \\
\text { by implementing different operating } \\
\text { conditions }\end{array}$ \\
\hline $\begin{array}{l}\text { Electron bunch } \\
\text { length at FELs }\end{array}$ & $\sim 1 \mathrm{~s}$ fs $-100 \mathrm{~s}$ fs & $\begin{array}{l}\text { Dependent on FEL and } \\
\text { optimized for experimental } \\
\text { needs } \\
\text { Bunch current maintained } \sim 1 \mathrm{kA}\end{array}$ & $\begin{array}{l}\text { Flexibility in achieving design goals } \\
\text { by implementing different operating } \\
\text { conditions }\end{array}$ \\
\hline $\begin{array}{l}\text { Normalized slice } \\
\text { emittance at FEL }\end{array}$ & $\begin{array}{l}<1 \mathrm{~mm} \cdot \mathrm{mrad} @ 1 \mathrm{nC} \\
\leq 0.5 \mathrm{~mm} \cdot \mathrm{mrad} @ \\
\leq 0.1 \mathrm{nC}\end{array}$ & $\begin{array}{l}\text { Lase at nm wavelengths with } \\
\text { low-energy beam } \\
\text { Requires low-emittance electron } \\
\text { source and low-emittance beam } \\
\text { transport }\end{array}$ & $\begin{array}{l}\text { Reduced costs from lower-energy } \\
\text { accelerator }\end{array}$ \\
\hline $\begin{array}{l}\text { Uncorrelated } \\
\text { energy spread at } \\
\text { FEL }\end{array}$ & $<200-300 \mathrm{keV}$ & $\begin{array}{l}\text { Lase at nm wavelengths with } \\
\text { low-energy beam } \\
\text { Energy spread impacts efficiency } \\
\text { of optical manipulations } \\
\text { Requires low-emittance electron } \\
\text { source and low-emittance beam } \\
\text { transport } \\
\text { Requires use of a laser heater to } \\
\text { control microbunching instability }\end{array}$ & $\begin{array}{l}\text { Reduced costs from lower-energy } \\
\text { accelerator }\end{array}$ \\
\hline Undulator period & $\leq \sim 1 \mathrm{~cm}$ & $\begin{array}{l}\text { Lase at } \mathrm{nm} \text { wavelengths with } \\
\text { low-energy beam }\end{array}$ & $\begin{array}{l}\text { Reduced costs from lower-energy } \\
\text { accelerator }\end{array}$ \\
\hline $\begin{array}{l}\text { Superconducting } \\
\text { undulators }\end{array}$ & $\begin{array}{l}\text { Planar and polarized } \\
\text { designs }\end{array}$ & No moving parts & $\begin{array}{l}\text { Reduced costs and improved } \\
\text { reliability }\end{array}$ \\
\hline Seeding & $\begin{array}{l}\text { Temporal coherence } \\
\text { approaching the } \\
\text { Fourier transform limit }\end{array}$ & $\begin{array}{l}\text { Seeded FELs offer close to } \\
\text { transform limit } \\
\text { Requires optical manipulations } \\
\text { of the electron beam }\end{array}$ & $\begin{array}{l}\text { High degeneracy, high energy } \\
\text { resolution }\end{array}$ \\
\hline
\end{tabular}




\section{Soft X-Ray FEL Technology R\&D}

\subsection{Injectors}

\subsubsection{Electron Gun}

The final performance of a FEL facility ultimately depends on the quality of the beam generated at the electron injector, in particular at the electron source (hereafter referred to as the electron gun). The design and optimization of the accelerator downstream of the gun can in the best case only preserve but not improve the beam quality obtained at the injector.

A number of requirements must be satisfied by an electron gun in order to operate in a highrepetition-rate FEL-based soft x-ray light source. Some of them, notably electron-beam brightness, are directly defined by the physics of the lasing process; others such as pulse duration and repetition rate, derive from the characteristics and needs of the experiments using the light. Finally, some requirements relate to the needs for ease of operation, cost minimization, and in particular reliability, which is fundamental if the gun has to operate in a user facility.

Table 2.1 summarizes and quantifies the minimal gun parameters that must be simultaneously met for the operation of a cost- and performance-optimized, high-repetition-rate soft x-ray FEL. This table was compiled accounting for users' needs [6] and benefited from theoretical and experimental study results obtained by groups working on FEL and injectors worldwide [7, 8, 9].

Table 2.1. Minimal electron guns requirements for a high-repetition-rate soft x-ray FEL

\begin{tabular}{|l|l|l|}
\hline \multicolumn{1}{|c|}{ Parameter } & \multicolumn{1}{c|}{ Range } & \multicolumn{1}{c|}{ Notes } \\
\hline Normalized emittance $(\mathrm{mm})$ & $\leq 1$ & $\sim \mathrm{nC}$ charge/bunch regime \\
\hline Normalized emittance $(\mathrm{mm})$ & $\sim 0.1$ & $\mathrm{pC}$ charge/bunch regime \\
\hline Accelerating field at the cathode $(\mathrm{MV} / \mathrm{m})$ & $\sim 20$ & \\
\hline Beam energy $(\mathrm{MeV})$ & $>0.5$ & \\
\hline Repetition rate $(\mathrm{Hz})$ & Up to10 & \\
\hline Vacuum pressure at cathode area (Torr) & $\sim 10^{-11}$ & $\begin{array}{l}\text { Required by high QE semiconducting } \\
\text { cathodes }\end{array}$ \\
\hline Bunch charge $(\mathrm{pC})$ & $\sim 1$ to 1000 & Depending on the FEL operation mode \\
\hline $\begin{array}{l}\text { Variable magnetic field at cathode region } \\
(\mathrm{T})\end{array}$ & up to $\sim 0.3$ & Emittance compensation and exchange \\
\hline
\end{tabular}

Several different gun technologies are available but not all of them simultaneously meet all of these requirements. For example, DC gun schemes using photocathodes can generate quality low-emittance beams, potentially operate at high repetition rates up to $\mathrm{GHz}$, can be compatible with magnetic fields at the cathode, and are capable of achieving the extreme vacuum required to operate "delicate" high-quantum efficiency semiconductor photocathodes with an acceptable lifetime. Unfortunately, obtaining the fields and energies of Table 2.1 in a DC gun pushes the 
technology to the limits and requires significant effort and expertise, especially in preventing insulator breakdown and field emission. Higher voltages in DC guns increase the complexity of the system; larger and difficult-to-build insulating ceramics are required, and the high-voltage parts of the system must be placed in inert gas tanks. Voltages of up to $\sim 350 \mathrm{kV}$ have been reliably generated [10], and there are groups working toward higher voltage [11], so far unsuccessfully.

Electron guns based on superconducting accelerator cavities are a promising technology that would allow for $\mathrm{CW}$ operation at high repetition rate up to $\mathrm{GHz}$, with accelerating fields potentially higher than in DC guns, and they are capable of extremely low vacuum pressures because of the effective cryo-pumping by the superconducting walls. A number of groups worldwide are pursuing the development of such sources [12], and metallic photocathodes operating at cryogenic temperatures have been demonstrated but with very low quantum efficiency. R\&D activity is now addressing techniques that would allow use of higher-quantumefficiency, high-temperature cathodes in superconducting structures. Cavity contamination by pollutants from photocathodes is a potential issue that could affect the cavity performance and that needs to be carefully investigated. Additionally, because of field exclusion by the superconducting walls (Meissner effect) magnetic fields in the cathode region cannot be easily applied. Such fields are necessary for emittance compensation as well as for some emittanceexchange schemes. Alternative schemes where the required magnetic field configuration is generated by exciting a specific high-order mode in one of the gun accelerating cells have been proposed and are under study [13].

A number of normal conducting guns operating in L-band ( $\sim 1$ to $2 \mathrm{GHz})$ and S-band ( $\sim 2$ to $4 \mathrm{GHz}$ ) have been developed and successfully operated in photo-injector schemes. A recent remarkable example is the LCLS gun at SLAC that is reliably operating beams with the brightness required for x-ray FEL lasing at $1.5 \AA$ [14]. Normal-conducting high-frequency guns are a reliable and mature technology with several important positive features. They have successfully achieved high gradients at the cathode $(>100 \mathrm{MV} / \mathrm{m})$ that allow running highcharge bunches with relatively short bunch lengths, and they are is compatible with a large number of cathode types and with the presence of magnetic field in the cathode area. On the other hand, owing to the high RF frequency, the power density on the cavity walls becomes significant when the cavity is operated at high gradients. This limits the maximum achievable repetition rate to $\sim 10 \mathrm{kHz}$ [15]. Additionally, the small RF wavelength does not allow for large pumping apertures and can therefore limit the overall vacuum performance.

The requirement for operating the FEL at high repetition rates [6] pushed gun designers to investigate schemes using normal-conducting RF structures with relatively low frequency (from $\sim 100$ to $700 \mathrm{MHz}$ ) $[16,17,18,19]$. As mentioned earlier, L- and S-band cavities are limited to pulsed operation by the maximum power density that can be dissipated on the cavity walls. On the other hand, lower-frequency cavities have lower accelerating fields than their normalconducting higher-frequency counterparts, but the fields are still substantially higher than in DC guns. As in the DC gun case, in order to control space-charge effects, the bunch length at the gun must be long enough to obtain the required upper limit in charge density. And, as with the higher-frequency normal-conducting RF cavities, these schemes are compatible with the presence of magnetic fields. 
Normal-conducting RF guns resonating in the VHF band (30-300 MHz) [18] experience a power density on the walls that becomes sufficiently small to be handled by conventional waterchannel-cooling techniques. Such schemes present remarkable simplicity and are based on mature RF and mechanical technology with promising performance in terms of reliability.

Additionally, because of the longer wavelength in these cavities, remarkably large apertures can be opened in the cavity walls allowing for high vacuum conductance when connected to vacuum pumping located outside of the cavity walls. With a VHF cavity, the azimuth of the cylindrical body can accommodate slots oriented in the direction of current flow in the cavity's accelerating mode. A significant fraction of the cavity surface area can be removed to provide connection between the cavity internal volume and a vacuum-pumping plenum connected directly to the outer circumference of the cavity. Extensive pumping in the plenum allow obtaining the low pressure in the cathode region needed for the operation of high-quantum-efficiency semiconductor cathodes, with long lifetimes.

Beam dynamics for the VHF photo-gun, with long pulse extracted over a relatively short fraction of the RF waveform, are similar to a DC gun. The cavity geometry is highly symmetrical, and perturbations of the TM-like accelerating mode are negligible. Production of low-emittance beams in this cavity has been shown in simulations [8], and are consistent with work on DC guns.

For these reasons, we choose a normal-conducting VHF gun to simultaneously meet the demanding performance requirements of a high-repetition-rate FEL facility, that are listed in Table 2.1.

\subsubsection{Cathode/laser-system choice}

One of the significant challenges for next-generation FELs is to achieve a high repetition rate, while maintaining or even reducing emittance below the levels currently reached during lasing. This imposes several new challenges for the injector, one of the main ones concerning the photocathode used for generating electron bunches. T he 1-MHz repetition rate for the proposed soft x-ray FEL facility is almost four orders of magnitude higher than the repetition rate for the LCLS, for example.

The LCLS gun uses a polycrystalline copper cathode, which in the gun achieves a quantum efficiency (QE) of approximately $5 \times 10^{-5}$ at the $255-\mathrm{nm}$ operating wavelength. The relatively low QE and the required short wavelength UV light results in significant demands on the laser system, even though the repetition rate is rather low. The FLASH FEL runs at $10 \mathrm{~Hz}$, but with a micro-bunch pattern that has 800 bunches in a macro bunch, for an average rate of $8 \mathrm{KHz}$. This performance is achieved with UV excitation $(265 \mathrm{~nm})$ with cesium telluride $\left(\mathrm{Cs}_{2} \mathrm{Te}\right)$ as the cathode material. This material has the great advantage that the QE is typically $5 \%$, but it is somewhat more fragile than the copper cathodes. Copper cathodes for the LCLS are prepared in UHV, cleaned, and then rapidly transferred into the gun in air, where after pump-down they are laser cleaned. The $\mathrm{Cs}_{2} \mathrm{Te}$ has to be prepared in UHV from deposition and reaction of cesium and tellurium, transferred to the gun, and then kept under UHV conditions at all times. These 
techniques have been developed over many years, and it now seems to be a mature and reliable technology. It has not however been tested at the repetition rates and average currents proposed here. The goal therefore is to test $\mathrm{Cs}_{2} \mathrm{Te}$ at high repetition rates and high average currents, but at the same time to develop a high QE photocathode that can operate with much longer wavelength light for excitation. This potentially alleviates many of the photocathode laser problems that might occur in high-average-power systems, and it may lead to a compact and reliable overall package.

\subsubsection{High-efficiency, long-wavelength photocathodes}

The choice in high-efficiency, long-wavelength photocathodes is really limited to cesiated GaAs, as typically used in DC guns, and alkali antimonide materials. The cesiation of GaAs with subsequent oxidation or fluorination leads to zero or slightly negative electron affinity. An electron photoexcited from the valence band will scatter and thermalize with the lattice during transport to the surface and escape across the material-vacuum barrier with high probability. Excitation is typically with photons in the range from the bandgap at $1.42 \mathrm{eV}(873 \mathrm{~nm})$ up to $2 \mathrm{eV}(620 \mathrm{~nm})$, and at the higher energies, QE well above $20 \%$ can be achieved. The disadvantage of these materials for FEL applications is that they must operate in extremely high vacuum; they suffer from saturation at high pulse charges; and they yield relatively long pulse lengths, caused by the long absorption length and the electron-phonon random walk to the surface. This problem can be partially solved at the expense of QE using thin epitaxial GaAs, and the charge-saturation issue can be improved by very heavy doping. The remaining problem is the extreme sensitivity of the surface to contamination and to ion-induced lattice damage. While highly suitable for very high-repetition-rate, low-pulse-charge applications, such as for an ERL, for an FEL we are seeking a more robust solution capable of very high bunch charges.

The alkali antimonides are a class of materials that have very high QE in the visible range of the spectrum [20]. The most commonly used types are $(\mathrm{Cs}) \mathrm{Na}_{2} \mathrm{KSb}, \mathrm{Na}_{2} \mathrm{KSb}$, and $\mathrm{K}_{2} \mathrm{CsSb}$ with emission thresholds of 1.4, 1.7, and $1.8 \mathrm{eV}$, respectively. Each has a peak QE of above 30\%, but the critical parameter is the QE at a value of photon energy for which the energy spread of the photoelectrons is still small. The reason is that for a source in which electrons are born in a random angular distribution, the thermal emittance is simply proportional to the product of the radius of the emission area of the cathode and the square root of the average electron energy. Higher photon energies result in higher QE but also higher thermal emittance. The thermal emittance cannot simply be reduced by reduction of the photocathode radius, because the minimum radius is set by space charge. Reduction of the radius beyond a certain value results in shielding of the accelerating potential in the gun (a Child-Langmuir limit for short pulses) [21]. These considerations lead to a value of "excess energy" in the photoemission spectrum of only about $0.5 \mathrm{eV}$ for acceleration gradients of $20 \mathrm{MV} / \mathrm{m}, \mathrm{nC}$ charge, and 1-mm beam radius. This in turn essentially sets the photon energy above threshold. This relationship is not linear, however, and depends on the details of the density of initial and final states. For ( $\mathrm{Cs}) \mathrm{Na}_{2} \mathrm{KSb}$, for example, a kinetic energy bandwidth of $0.5 \mathrm{eV}$ corresponds to a photon energy of $2.2 \mathrm{eV}$, i.e., $0.8 \mathrm{eV}$ above threshold, for which the QE is around 20\% [22]. The required photon energy would be modified, however, by the Schottky lowering of the surface barrier height by the accelerating field gradient.At $20 \mathrm{MV} / \mathrm{m}$, this amounts to $0.17 \mathrm{eV}$, resulting in a required photon energy of $2.03 \mathrm{eV}$. If we assume that we are using a frequency-doubled ytterbium-based laser $(2.37 \mathrm{eV})$, then the resulting kineti- energy bandwidth and hence thermal emittance would be too high. Use 
of $\mathrm{K}_{2} \mathrm{CsSb}$, however, would give close to the exact bandwidth required and result in over $10 \%$ QE. The electron affinity can be adjusted slightly by small changes to the stoichiometry of the material and by oxidation.

The main challenges associated with these materials relate to details of the growth mode and stoichiometry and to their robustness in the vacuum and high-field conditions in the photocathode gun. There is a prior example, however, of their use in FEL technologies, for example in the Boeing-Los Alamos high average power visible-IR FEL [23]. In that case, in a 433-MHz cavity, high average currents at bunch charges of over $5 \mathrm{nC}$ were demonstrated. The main technical issue affecting their use was their sensitivity to oxygen and water, a problem that was to some extent alleviated by improvements to the vacuum system. With the more open pumping geometry of low-frequency RF cavities and better UHV technology available today, it is expected that vacuum issues should not be significant in determining cathode lifetime.

One significant advantage of the use of multi-alkali antimonides is that they can be used with relatively low-average-power green lasers, derived from ytterbium fiber or thin-disk laser technology. For example, assuming a 1-nC charge requirement, 10\% QE, 532-nm wavelength, and $25 \%$ conversion efficiency from 1064 to $532 \mathrm{~nm}$, then approximately $100 \mathrm{~nJ}$ pulse energy at $1064 \mathrm{~nm}$ will be needed. There would be additional power needed owing to losses associated with pulse shaping in time and space, but in the IR and the green wavelength regions these losses are typically small. Allowing a factor of 10 for these losses and for operation at less than optimum QE means that the pulse energy would be $1 \mu \mathrm{J}$, and the average power would be $1 \mathrm{~W}$ for $\mathrm{MHz}$ operation. This is in the realm of high-power thin-disk oscillators or of low power amplified disk or fiber lasers. Such systems have been developed extensively for a range of industrial applications and are likely to be a robust basis for development of laser systems tailored to this application.

\subsubsection{Proposed injector R\&D activity}

The injector R\&D activity will involve two main parts, the first dedicated to the VHF gun development and test, and integration of the VHF gun with the other injector components required to control the beam properties and to match the injector to the FEL main linac. The second part of the program will involve testing of cathodes in the VHF gun. Both activities will be undertaken simultaneously.

\subsubsection{Electron-gun development}

In the first stage of gun R\&D, we will verify the capabilities of the VHF cavity including the ability to produce 1) the required voltage and accelerating fields in $\mathrm{CW}$ mode, and explore voltage limits from multipacting or other causes (i.e. full power RF test); 2) the ultra-high vacuum performance required to operate high quantum efficiency cathodes with a reasonable lifetime ( $\sim 10^{-11}$ Torr); and 3) extraction of bunches up to $1 \mathrm{nC}$ with $\mathrm{MHz}$ repetition rate. This last task will involve exploration of different types of cathodes and laser requirements. A lowenergy diagnostic beam line will be designed and constructed to measure the most basic beam parameters (a Faraday cup to measure charge, a virtual cathode to image the laser pulse, screen to image dark current, etc.) at the gun exit and to characterize the performance of the photocathode-laser system. 
Following demonstration of the fundamental cavity performance and initial tests of beam from the gun, the VHF gun will be integrated into a beamline to allow more complete beam measurements. The beamline will include magnetic components required for beam transport and emittance compensation, a "buncher/booster" accelerator required to longitudinally compress and bring the beam energy to several tens of $\mathrm{MeV}$ and to allow for full emittance compensation, and a comprehensive diagnostic beam line to fully characterize the beam properties at that energy. Diagnostics and instrumentation will include the "standard" set of current, position and profile monitors plus a transverse deflecting cavity and a spectrometer that will allow for phasespace-sliced measurements in either longitudinal or transverse planes.

It is important to remark that, while the cavity-cathode-laser systems must be tested at the full repetition rate, these may be demonstrated at the extraction energy of the gun. The full characterization of beam parameters, carried out at higher energy, can be made at a much lower repetition rate in order to simplify the system and reduce the costs of the R\&D program. We plan to use a pulsed low-repetition-rate normal-conducting system for the buncher-booster and deflecting cavity for slice emittance measurements.

The final performance demonstration will involve a number of intermediate steps and tests that include building prototypes of critical parts of the cavity (braze tests, surface finish tests, etc) for defining and optimizing RF, vacuum and mechanical fabrication techniques; simulations of multipacting and its mitigation in order to avoid dangerous resonances in the range of operation; careful design and, potentially, conditioning of components in regions of high electric field to minimize field emission and dark current; and simulation and analytical analysis of ion and electron back-bombardment of the cathode.

Beam-dynamics simulations are required for defining the complete injector layout, including accelerating sections, beam diagnostics, and matching beamlines. Extensive and detailed beamdynamics simulations must be performed to design experiments and allow comparisons of experimental results with expectations.

Of fundamental importance is the R\&D on the laser-cathode photoemission system and the capability of controlling the shape of the laser pulse distribution. The final performance of the gun dramatically depends on such systems, and the proposed R\&D activities are described in the next subsection.

In summary, the proposed gun $R \& D$ activity will be articulated in three years and organized according to the following steps:

a) VHF structure and full RF test. This part includes the following tasks:

- Design and fabrication of the VHF cavity

- Procurement of the CW RF power source for the gun cavity

- Design and fabrication of the RF power-distribution line

- Preparation of the testing area and installation of the cavity, RF source, and RF power distribution. 
- Design, fabrication, and installation of the low-level RF system

- Completion of low and full power RF test of the gun.

b) Cathode testing setup and photocathode-laser system tests:

- Design and fabrication or procurement of a vacuum load-lock system for the preparation and easy replacement of cathodes

- Verification of the ultimate vacuum performance of the gun

- Design and fabrication of a diagnostic beamline for use in characterization of the gun-cathode-laser systems at the operational energy of the gun and up to full repetition rate $(\mathrm{MHz})$.

- Installation and characterization of the selected laser-photocathode system

- Characterization of the laser longitudinal and transverse pulse-shape system

- Characterization of the electron beam and photocathodes at the operational energy of the gun for different charge and bunch distributions and comparison with simulation results.

c) Higher-energy beamline and beam-parameter characterization. The tasks include:

- Definition and purchasing of the normal conductive RF buncher-booster system, including the RF power source

- RF conditioning of the buncher-booster system Design, construction, and installation of the high-energy diagnostic beamline

- Design, fabrication (or purchasing) and installation of a transverse deflector cavity, including the RF power system

- RF conditioning of the transverse deflector system

- Commissioning of the injector with full characterization of the beam parameter at the booster exit at the repetition rate allowed by the buncher-booster system.

\subsubsection{Proposed R\&D on cathode-laser system}

The proposed development program on multi-alkali antimonides photocathodes will be centered on the following areas:

a) Design and build deposition systems

- Optimize the deposition process and the chemistry of antimonide formation

- Understand the micro-crystallinity and growth modes

- Integrate standard UHV thin film deposition techniques with in-situ XPS, UV-PEEM and x-ray or electron diffraction.

b) Characterization of cathodes during and after deposition

- Measure QE at the operating wavelength

- Measure wavelength dependent QE

- Measure optical and photoconductivity properties 
- Measure kinetic energy distribution, as a function of emission angle as a way of determining the transverse momentum spectrum. Typically this is done using time-offlight imaging electron spectroscopy

- Measure the sensitivity of the QE and kinetic energy spectrum to the details of the cathode stoichiometry and oxidation state, by variation of the deposition conditions.

c) Operation and damage studies

- Measure the sensitivity of the cathode materials to laser illumination, high accelerating fields, to contamination, and to accumulated extracted charge. Some of these studies could be performed in a deposition chamber, but this work would mainly be carried out in the high-power RF cavity discussed in Section 2.1.3.1.

Owing to the UHV characteristics of VHF RF guns and the large advantages of multi-alkali antimonides over other materials, these materials will be studied first. It is, however, prudent and part of this R\&D proposal to also study the properties of cesium telluride as a backup to the antimonides. The ultimate decision of which photocathode to use will be based on assessments of laser requirements and technology, QE, robustness and ease of manufacture and use.

Beam dynamics studies and experimental work by many groups around the world showed the importance of controlling the bunch-distribution shape at the cathode to minimize emittance growth due to space charge effects. In photo-injectors, this control can be conveniently achieved by shaping the laser pulse that generates the photoemission. For this pre-conceptual design $R \& D$, we only require a basic laser system for cathode testing. This would be based on a commercial 1$\mathrm{MHz}, 2-\mu \mathrm{J}$, picosecond , 1064-nm fiber laser, which would be frequency-doubled. We would use this system for assessments of methods for transverse-profile modification and temporal pulse shape control, as well as for all the basic commissioning of the photo-injector. Part of this proposal is also to test and develop techniques to shape and control the laser-pulse distribution in both the transverse and longitudinal dimensions.

\subsection{FEL Design}

For production of highly coherent and well-controlled x-ray beams, the 6D phase space of the electron beam at the radiation source must be precisely tailored. The electron bunch goes through an elaborate process that prepares it for use in an FEL. The emittance, current, and energy spread in the electron bunch must be controlled and preserved from production at the cathode, through the bunching and accelerating processes, and transport to the photon-production devices of the facility. The most essential part of the process is acceleration. One needs high electron energy to obtain short-wavelength radiation (see Section 1.3), and in Section 2.3 we discuss how one can lower the requirement for the electron-beam energy by reducing the undulator period. An additional requirement is achieving high peak current and preserving the small emittance obtained in the gun because efficient lasing in FEL requires electron beams with a high brightness (see Section 1.3). A multitude of interactions between the charged-particle beam and its environment can degrade the beam brightness, and control of these processes is essential. We classify the acceleration as a technology issue (discussed in Section 2.4) and brightness as a beam-dynamics issue further discussed here. 
When the intense electron bunch propagates down the accelerator, it is subject to various collective effects. These include space-charge effects, which can be further divided into (a) longitudinal space-charge effects (LSC) that continue to play a visible role in the formation of microstructures inside the electron bunch even at high electron beam energy and (b) transverse space $=$ charge effects that affect electron-beam emittance at relatively low electron beam energies. Longitudinal wakefields produced by the accelerating structures can produce nonlinear variation of the slice energy along the bunch. Transverse wakefields can significantly increase projected emittance. Also relevant for beam stability is the emission of coherent synchrotron radiation (CSR) in the bunch compressors and in the electron-beam switchyard leading to the FELs. For a perfectly smooth bunch without any internal microstructure, the effect of the CSR is very similar to the effect of the longitudinal wakefields, but if there are density modulations inside the electron bunches, the CSR tends to enhance them, thus giving rise to the so-called microbunching instability. In general, this instability is driven by a combination of LSC and CSR and can produce significant fragmentation of electron bunches, forcing electrons to occupy a larger area in the longitudinal phase space and thus degrading electron-beam brightness. The nonlinear curvature of the RF waveform and the nonlinear time-of-flight characteristics of the bunch compressor are often responsible for the appearance of large spikes in the peak current at the head and tail of the electron bunches. The spike at the head of the bunch can induce resistive wall wakefields that can cause a nonlinear energy variation along the electron bunch during its motion through the spreader and FEL undulator. The spike at the tail of the bunch can also induce a nonlinear energy variation along the bunch that is due to the CSR in the electron beam switch yard.

All the above-listed collective effects are now rather well understood and described in the literature. Computer codes such as LiTrack [24], elegant [25], CSRTrack [26], IMPACT [27], Vlasov solver [28] were developed specifically to address beam-dynamic issues and to guide machine designers in their choices for the machine configurations. These codes were benchmarked against theory, between themselves, and wherever possible against experiments [29], for example most recently during the commissioning of the LCLS [30]. One specific instrument, i.e., the laser heater, was tested for the first time ever during recent LCLS commissioning. The laser heater is essential for suppressing the microbunching instability by increasing the uncorrelated energy spread in the beam at low energy. While it was proved to perform in good agreement with the anticipated performance [31], there is need for further exploratory studies of this instrument during machine-development studies at the LCLS. In addition, development of high-resolution temporal and spectral diagnostics is critical to the detailed understanding of the longitudinal-phase-space structures of the electrons. Such a detailed understanding is essential for seeded FELs and can be tested using the high-brightness LCLS beams.

As is often the case, even if all components are known in principle, the design of the entire machine remains a challenging undertaking. The difficulty arises when one attempts to develop a cost-optimized design with low technical risk. One area to be addressed is the electron-beam switchyard, which distributes electron bunches between the FELs. A compact, flexible, and costeffective solution requires a high-voltage, high-repetition-rate pulsed kicker. Although a pulsed power supply with required repetition-rate and voltage-pulse capabilities is commercially available, its reliability and long-term pulse-to-pulse stability must be demonstrated and 
improved if needed. This would require purchasing a stripline and a pulser and testing it with electron beam.

Another subject for study is electron-beam-tail management and halo confinement. Being able to remove stray and unwanted electrons as early as possible is rather critical for a machine with a high average electron-beam current. Here, developments in techniques to collimate the electron beam are required, in order to accommodate the high power of the beam in a high-repetition-rate facility. At the pre-construction stage, this will involve theoretical and simulation studies to develop design concepts.

In order to obtain high peak current, one compresses the electron bunches produced in the electron gun by creating a linear energy variation along the electron bunch, i.e., an energy chirp, and then sending this bunch through a dispersive magnetic chicane. As a result, the electron bunch emerges from the chicane with an energy chirp, which is undesirable for FEL operation. In the case of LCLS and FERMI@Elettra linacs, this energy chirp is removed by the electron bunch acting on itself via the longitudinal wakefield $[32,33]$. However, in the TESLA type supeconducting linac, for example, the wakefield is weak and often is not sufficient to remove the chirp. Thus "de-chirping" of short electron bunches requires running the electron bunch offcrest on the RF wave, which is a rather ineffective use of the linac. In principle, a short piece of a small diameter ceramic vacuum chamber could be used to create the needed wake. Theoretical studies of this alternative approach could result in a cost-effective solution of the problem.

Section 2.3 discusses the R\&D plan for development of undulator technologies that may have a significant impact on the cost of the entire soft x-ray FEL facility. We highlight here the need for characterization of the short-range wakefields induced by an ultrashort electron bunch in a narrow-gap vacuum chamber that would be a part of technology requirement if short period undulators are to be used. Theoretical and experimental studies for both warm and cold vacuum chambers will lead to understanding of the impact of the wakefield on FEL performance.

The leading candidate for a seeded FEL is the echo-enabling harmonic-generation (EEHG) technique [34]. Success of this technique is critical to the ability to produce temporally coherent X-rays beyond the short-pulse capabilities of SASE and without major advances in laser technology for seeding. Recent studies [35] indicate that by using this technique, one can reach $\mathrm{x}$-ray wavelengths in the water window beginning with an optical seed in two relatively simple acts of electron-beam manipulation by the laser. There is high confidence in our ability to perform these manipulations, based on previous experience in producing electron-beam-energy modulation and microbunching via interaction with the laser [36, 37] and on numerical studies using computer codes such as GENESIS [38], GINGER [39], and other codes dedicated to FEL simulations. These codes have more than a decade-long history of development and were benchmarked between themselves and against the experiment at FLASH [40] and recently at the LCLS [41]. However, the preservation of fine structures in the beam that support coherent radiation at nanometer wavelengths deserves further study. The smearing of fine structure during transport from the modulator to the radiator caused by second-order transportation effects needs to be addressed both theoretically and experimentally. 
We propose rapidly pursuing proof-o- principle experiments of EEHG, followed by more detailed studies to fully characterize the process and develop techniques. Generation and preservation of the fine structures can be performed at beam energies of $\sim 100 \mathrm{MeV}$ to demonstrate the fundamental physics behind the echo-enabling microbunching technique. At this relatively low energy, only relatively low-harmonic generation can be demonstrated initially.

The issues involved in extending the technique to the $100^{\text {th }}$ harmonic to produce photons in the $\mathrm{keV}$ energy range can be further explored under future proposals, at future facilities with the required beam energy and density. Microbunching-destroying CSR effects, space charge and path length debunching effects will be studied. Confirmation of the theory for the keV photonenergy range is an essential step towards the realization of an echo-seeded x-ray FEL.

There is somewhat high anticipation in the scientific community that soft x-ray FELs producing sub-femtosecond pulses can be used to investigate how chemical bonds in molecules form, break or change [42]. The ability for generating sub-femtosecond $\mathrm{x}$-ray pulses synchronous to a short external-stimulus light pulse has been demonstrated so far theoretically (see [43] and references therein) but not experimentally. Most approaches focus on using a few-cycle laser pulse with carrier-envelope-phase stabilization to produce a characteristic energy modulation of the electrons via the laser beam-electron beam interaction in a one- or two-period wiggler magnet. Lasers with the required pulse energy are now commercially available, and the wiggler magnet is a rather standard design. Practical implementation would require assembling the entire system, which will also include diagnostics for characterization of the laser beam-electron beam interaction upstream of the line of the FEL undulators. For example this system may be implemented at an existing or under-construction FEL facility. In addition to acting as the test bench of the technology, this syste, will stimulate the development of diagnostics for the characterization of the sub-femtosecond x-ray pulses.

Other approaches to seeding need to be explored by modeling and experiment, to gain experience of optical-manipulations techniques. Development of codes to accurately model seeding of the electron bunch and the subsequent radiation of the seeded bunch in an FEL will benefit FEL designs. Experiments in seeding of an FEL, with characterization of the seed laser performance and the FEL output, including power, temporal and spatial coherence, tunability, and shot-to-shot stability, are required, together with comparisons of experimental results with simulations. Simulations and experiments may involve HGHG using a UV seed, harmonic cascade FELs to reach into the water window, HHG systems to produce a shorter-wavelength seed, and ultrafast pulses. Understanding gained will be used to further develop FEL designs to reduce risk in a future facility.

In the soft x-ray wavelength region, polarization control (from linear to circular) is highly desirable in studying ultrafast magnetic phenomena and materials science [44]. Variable polarization could, in principle be provided by an APPLE-type undulator. However, its mechanical tolerance for lasing at x-ray wavelengths has not been demonstrated, and its focusing property may change significantly when polarization is changed. An alternative approach for polarization control is the so-called "crossed undulator" $[45,46]$, which provides not only polarization control but also fast polarization switching with a pulsed-phase-shifter chicane. This scheme may be tested at existing or planned FEL facilities by adding a phase shifter and a short 
vertically polarized undulator after the FEL reaches saturation in the main undulator, in order to verfiy it can provide flexible polarization for the x-ray pulses.

Summarizing the above discussion we identify the most critical R\&D goals requiring pre-project support:

- Demonstrate the EEHG technique in experiments using electron beam available at low energy $(\sim 100 \mathrm{MeV})$. Measure the harmonic output of a bunch seeded at $\sim 800 \mathrm{~nm}$ and $\sim 1600 \mathrm{~nm}$, and compare to simulations. Study microbunching-destroying CSR effects and space-charge and path-length debunching effects, in order to understand the preservation of fine energy structure in high-brightness beams.

- Participate in the design of experiments in seeding of an FEL using optical seed lasers. Simulations, requiring some code development, will be used to model performance. Experiments may be performed at existing facilities and facilities under construction worldwide. Experiments may involve HGHG, harmonic-cascade, and HHG-seeding techniques and will characterize seeded-FEL performance, including coupling the seed to an electron bunch (generated by a conventional or laser-plasma-based accelerator), power output, temporal and spatial coherence, tunability, shot-to-shot stability, and reliability. Compare experimental results with simulations for a range of seeding conditions to explore effects affectomg performance. Design of seeded-FEL configurations for soft xray applications.

Also critical but the subject of future proposals will be further exploration of seeding and particularly extension toward the soft x-ray wavelengths.

Other activities that will require study under project or other R\&D funds are:

- Demonstrate long-term reliability and pulse-to-pulse stability better than $10^{-3}$ for a pulser loaded by a stripline operating at 10 -to- $15 \mathrm{kV}, 10 \mathrm{~ns}$, and $100-\mathrm{kHz}$ repetition rate. These sensitive measurements are best performed using an electron beam.

- Design an electron-beam-shaping and collimation system to remove front and back longitudinal tails from a high-power ( $1 \mathrm{~mA} \mathrm{CW})$ electron beam.

- Design a wakefield-inducing component to control the energy chirp along the electron bunch and test it with electron beam. The wakefield should be adjustable to allow flexibility in control of bunches of different charge distributions.

- Design and test a system for attosecond x-ray pulse generation.

- Validate theory and modeling of microbunching and laser-heater performance.

- Design concepts for high-resolution temporal and spectral diagnostics with micron spatial resolution.

- Design systems for x-ray polarization control using the "crossed undulator" approach allowing fast polarization switching with a pulsed phase shifter chicane. Determine performance parameters. 


\subsection{Insertion Devices}

The general design goals for undulator insertion devices in synchrotron rings, FELs, and ERLs are (a) to build coherence in the x-ray pulse by synchronization of photon production over the entire undulator length, and (b) to provide the means for producing the requisite spatially periodic magnetic field having appropriate characteristics (strength, period, polarization capability), while maintaining coaxial alignment of the electron-beam path with the undulator axis (and with, in some cases, a co-propagating laser beam). The performance characteristics of these light-producing undulators depend on a variety of factors, including, among others, electron energy, undulator period, and field profile. The photon energy, tuning range, and polarization characteristics of an undulator are a function of these three parameters, with tuning range provided by varying the magnetic-field strength. The current state-of-the-art (SOA) insertion devices include planar in-vacuum undulators for linear-polarized radiation and elliptical permanent-magnet (PM) undulators for variable polarization. Manufacture of these devices is now well understood, and a large number of these devices are installed and functioning at light sources around the world.

To optimize future FEL facilities, beyond-SOA capabilities are needed. Short-period, small-gap undulators can provide access to shorter-wavelength radiation, and high magnetic field is often also required to provide the desired spectral range. Indeed, such developments will play a critical role in achieving optimal accelerator systems design, performance, and cost. Undulator parameters determine the electron-beam energy required to radiate at a given wavelength, and thus determine accelerator length and cost as well as the required undulator length and cost. As a result, ultimate undulator performance possibilities and capabilities need to be understood very early in the facility design stage.

A variety of beyond-SOA undulator concepts have been proposed. Offering the most promise are the general class of superconducting undulators (SCUs), including NbTi-based, $\mathrm{Nb}_{3} \mathrm{Sn}$-based, and high temperature superconductor (HTS)-based designs. Encompassing both linear and elliptically polarized radiation capabilities will be an important aspect of future designs. Modeling and first prototype demonstrations suggest planar SCU's can yield a 40\% increase in field over SOA undulators. While some elliptical SCU schemes yield field performance equivalent to that of SOA EPU's, they can have dramatically enhanced spectral range, which is achieved by the use of the "period-doubling" obtained by selectively exciting coils in the device. Other beyond-SOA undulator technologies have also been proposed, including smaller bore in-vacuum permanent magnet (PM) designs, cryogenic PM designs, and more exotic, including microwave, $\mathrm{RF}$, or pulsed undulator concepts.

A common thread in all these proposed technologies is that they offer the potential for either (a) vastly enhanced radiation properties (intensity, energy, and/or polarization) or (b) drastically reduced new facility cost, or (c) both. Preliminary estimates indicate that new beyond-SOA undulator concepts offer a potential in reduction of undulator costs of $25-40 \%$ (SC vs PM, depending on configuration). Moreover, the beyond-SOA undulator concepts also offer a similarly significant reduction in cost resulting from a shorter linac beingrequired to deliver a lower-energy electron beam. 
R\&D is needed to validate the feasibility of these new undulator concepts. To minimize cost and optimize performance of new facilities, ultimate undulator performance capability, compatibility, and design need to be understood early in the facility design stage. For new FEL facilities, R\&D aimed at eventual reduction-to-practice of these beyond-SOA undulator technologies offers a low-cost path to significant performance enhancement and capability. Specifically, undulator R\&D work is needed in the following areas, in order of priority:

1) Device prototyping: HTS-tape, bifilar helical, SC-EPU

- Prototype small bore ribbon/tape HTS-based linear polarized SCU technology design

- Validate robustness features, including intermediate liners

- Validate feasibility of very inexpensive and suitable design for small-gap FELs

- Prototype proposed bifilar helical $\mathrm{NbTi}$-based or $\mathrm{Nb}_{3} \mathrm{Sn}$-based $\mathrm{SCU}$ design

- Prototype proposed adjustably polarizing layered bifilar helical SCU design

- Prototype proposed elliptically polarizing SCU design (SC-EPU)

○ Determine maximum polarization switching rates

- Validate cold switching of modes for varying periodicity in SC-EPU designs

$\circ$ Evaluate alternative methods to yield fast polarization switching.

2) Measurement system (prototype-scale)

- Design, develop SCU-appropriate (e.g. cryogenic) undulator-field-measurement schemes

○ Validate the adequacy of local (Hall-probe, pulsed wire) measurements for trajectory correction and maintaining beam-laser synchronicity

- Validate the adequacy of field-integral measurements to quantify global beam steering .

3) Prototype shimming techniques for trajectory, phase, and synchronicity

- Prototype proposed cryogenic phase-error and steering trim/tuning designs

- Develop small-bore intra-sectional undulator positional-field-correction schemes (tuning).

4) Study trajectory, phase, and synchronicity error-analysis and tolerance

- Develop undulator design with FEL accelerator physics and synchronicity requirements

- Develop inter-sectional undulator field and device alignment capability and schemes.

5) System optimization: electron energy, gap and period, tuning, polarization

- Determine adequacy of undulator mechanical and magnetic tolerances; perform error analyses

- Determine adequacy of polarization-dependent optical properties of candidate undulator technologies.

6) Wakefield effects: operating gap

- Investigate and mitigate surface-image-current-induced wakefields on beam bunches in small-bore devices. 


\subsection{Linac Systems}

\subsubsection{Linac parameters}

Table 2.2 lists the parameters for the CW SC linac.

Table 2.2 Parameters for the CW SC Linac.

\begin{tabular}{|l|c|}
\hline Linac Initial Energy & $10 \mathrm{~s}$ of $\mathrm{MeV}$ \\
\hline Linac Final Energy & $2.5 \mathrm{GeV}$ \\
\hline Gradient & $15-20 \mathrm{MV} / \mathrm{m}$ \\
\hline Bunch Charge & $1 \mathrm{nC} \mathrm{max}$ \\
\hline Bunch Spacing & $1 \mathrm{us}$ \\
\hline Current & $\begin{array}{c}\text { Up to } 1 \mathrm{~mA} \text { average } \\
1 \mathrm{kA} \text { peak }\end{array}$ \\
\hline Bunch length & $1 \mathrm{ps} \mathrm{down}$ to $\sim 1 \mathrm{fs}$ \\
\hline $\begin{array}{l}\text { Normalized bunch } \\
\text { Emittance }\end{array}$ & $\sim 1 \mathrm{~mm} \bullet \mathrm{mrad}$ \\
\hline
\end{tabular}

\subsubsection{Cavity design}

For a CW linac of this size, one of the design drivers is the cryogenic heat load from both the fundamental accelerator mode and high-order monopole modes. For the fundamental mode, one wants to choose a cavity frequency (f) at which there is a high shunt impedance per unit length (r), as the cryo-loading scales as the inverse of this quantity for a fixed gradient. Below a certain frequency, when the residual surface resistivity dominates the Bardeen-Cooper-Schrieffer (BCS) resistivity, $r$ scales as $\mathrm{f}$. Above this frequency, when the BCS resistivity dominates and increases as frequency squared, $r$ scales as $1 / f$. Thus the maximum value of $r$ occurs roughly at this transition frequency.

In designing their 100-mA, 20-MV/m ERL, Cornell considered such a tradeoff. Assuming a "moderate" 10-nOhm residual resistively, they computed that their minimum AC power for $2 \mathrm{~K}$ cooling would occur with a cavity frequency of $1.2 \mathrm{GHz}$ (see Figure 1 in [47], and Figure 2 in a more recent paper, [48], where $7 \mathrm{nOhm}$ was assumed). However, DESY has measured resistivities as low as $3 \mathrm{nOhm}$, which would lower this optimal frequency to around $800 \mathrm{MHz}$, where the Spallation Neutron Source (SNS) cavities operate. This may not be practical however, and SNS has in fact measured a residual resistivity of $7.9 \mathrm{nOhm}$ in one of their cavities [49]. However, the SNS frequency choice was also driven by other issues such as the desire for a large iris opening to avoid proton losses, and by requirements associated with the lower beta $(0.61$ and $0.81)$ cavities they use. In polling various experts, a residual resistivity of 5-10 nOhm seems achievable, and if lower values were achieved relative to that optimal for the frequency choice, one could always lower the operating temperature somewhat below $2 \mathrm{~K}$ to exploit this (this is explored in [47]), but the cryogenic system would become more difficult to operate. 
For the higher-order monopole modes, the total non-resonant losses scale as the inverse of the iris radius squared, so in general lower frequencies are preferable. Including the cryo-loading from these losses, Cornell finds that their minimum AC power for $2 \mathrm{~K}$ and $80 \mathrm{~K}$ cooling [the latter for the higher-order-mode (HOM) absorbers] occurs at $1.1 \mathrm{GHz}$ [48]. However, they chose $1.3 \mathrm{GHz}$ where the TESLA collaboration has developed cavities and a variety of RF sources are available. The higher-frequency choice was also motivated by the somewhat lower cost due to the smaller cavity size and the lower associated bunch charge, which would help preserve their low bunch emittances. However, Cornell does not plan to use the standard TESLA 9-cell cavity design, as their high beam current requires modifications to improve the HOM damping. Instead they developed a seven-cavity design with six HOM loop couplers (although the number has been scaled back in more recent designs) and a larger beam tube on one end to reduce mode trapping. They also include an $80 \mathrm{~K}$ ferrite broadband ring-absorber after each cavity to remove the $140 \mathrm{~W} /$ cavity of HOM power efficiently (for ILC/XFEL, only one such absorber is used per eight- or nine-cavity cryomodule).

For the soft x-ray linac, the beam current would be much lower than that in the Cornell ERL ( $1 \mathrm{~mA}$ versus $200 \mathrm{~mA}$ including the de-accelerated beam), although the non-resonant HOM losses are still fairly large because of the high charge per bunch (up to $1 \mathrm{nC}$ versus $77 \mathrm{pC}$ for the ERL). Scaling by the bunch frequency and bunch charge squared yields HOM losses equal to 7\% of the ERL case (or $9 \mathrm{~W}$ per cavity). For the ILC, the losses are only $0.47 \%$ of the ERL case (or $0.6 \mathrm{~W}$ per cavity). However, the low bunch frequency (1 MHz versus $2.6 \mathrm{GHz}$ for the ERL) makes the soft X-ray FEL linac much more susceptible to monopole-mode resonances, and short bunch operation (tens of femtoseconds versus 2 ps for the ERL), will mean much more of the HOM power will be at high frequencies where is it harder to attenuate.

Based on these considerations, it would be best to start with the 1.3-GHz Cornell cavity design and reexamine the HOM issue for the soft X-ray FEL linac to see if a more ILC-like design could be used. The main goal would be to make sure the $9 \mathrm{~W}$ per cavity of HOM power spread over a large frequency range would be dissipated mainly in absorbers at $80 \mathrm{~K}$, something that is currently being examined for the ILC. To put this in perspective, the power dissipated at $2 \mathrm{~K}$ in a cavity with unloaded $\mathrm{Q}\left(\mathrm{Q}_{\mathrm{o}}\right)$ of $1.5 \times 10^{10}$ and a $20-\mathrm{MV} / \mathrm{m}$ gradient is $29 \mathrm{~W}$, so it could increase significantly if a large portion of the HOM power is also dissipated at $2 \mathrm{~K}$ (as in the Cornell ERL Linac, it is assumed that there would be no warm sections between the cryomodules in the linac as there is at SNS).

If the $805-\mathrm{MHz}$ SNS cavity frequency were used instead, the HOM power would be reduced by about $60 \%$, and as noted above, the fundamental mode losses would be smaller at $2 \mathrm{~K}$ if a low residual resistivity can be achieved. However, the sustainable gradients are likely to be smaller than in TESLA-like cavities, if the SNS linac performance is an indication. That is, their beta $=0.81$ cavities operate in the $10-15 \mathrm{MV} / \mathrm{m}$ range [50], and if one corrects for the higher surface-to-accelerator fields due to the low beta, this translates to $11-17 \mathrm{MV} / \mathrm{m}$ gradients for beta $=1$ cavities, which is marginal. The lower gradients relative to that achieved in TESLA-like cavities (typically $>20 \mathrm{MV} / \mathrm{m}$ ) may be related to the larger surface area of the cavities. 


\subsubsection{Cryogenic load}

For $1.3-\mathrm{GHz}$ cavity operation at $2 \mathrm{~K}$, the overall cryo-load is modest. Scaling the TESLA cryoload design values to the soft x-ray FEL linac, the total AC power for cryo-cooling would need to be $3.0 \mathrm{MW}$, assuming a unity overcapacity factor, a gradient of $20 \mathrm{MV} / \mathrm{m}$, and an average cavity $Q_{0}$ of $1.5 \times 10^{10}$. This $Q_{0}$ value is higher than the $1 \times 10^{10}$ value assumed for the ILC but $\mathrm{Q}_{\mathrm{o}}$ decreases at the higher ILC gradient of $31.5 \mathrm{MV} / \mathrm{m}$. It may be somewhat optimistic, but six recent ACCEL cavities processed at JLab had a mean $Q_{o}$ equal to this value at $20 \mathrm{MV} / \mathrm{m}$.

Of the total AC power, about $80 \%$ is required for the dynamic cavity load, $6 \%$ is for RF leakage in the non-SC $2 \mathrm{~K}$ beam-pipe region, $3 \%$ is for the $80 \mathrm{~K}$ heating in the power couplers (one per cavity) and only $0.6 \%$ is for the $80 \mathrm{~K} \mathrm{HOM}$ absorbers. The AC power for the cavity cryo-load is similar to the Cornell ERL estimate when scaled for machine energy. The power estimate assumes "state-of-the-art" efficiencies with the plant operation a full capacity (i.e., there is little room for improvement using the "Ganni" cycle compression scheme, for example, which helps when running cryogenic systems below capacity).

\subsubsection{Cryomodules}

The ILC/XFEL cryomodule design with eight cavities each should be able to handle the higher heat load in the soft $\mathrm{x}$-ray FEL linac with only small modifications. Basically, the tube from the helium vessel to the $2 \mathrm{~K}$ 2-phase pipe would need to be increased somewhat (from and ID of 55 $\mathrm{mm}$ to about $64 \mathrm{~mm}$ ). The 72-mm ID, 2-phase superfluid helium distribution pipe should still be acceptable, with a pressure drop of $0.18 \mathrm{mbar}$ and $4 \mathrm{~m} / \mathrm{sec}$ flow before it connects (via a 72-mm ID 2-phase pipe) to the $300 \mathrm{~mm}$ gas return line (which also serves to support the cavities and quadrupole magnets). With about 13 grams/sec of helium added at each cryomodule, the pressure drop in the gas-return pipe by the end of the $\sim 20$ cryomodule linac would be only be a few percent of the 30 mbar helium pressure, which should also be acceptable (at lower temperature, the gas-return pipe would need to be more finely segmented). Also, the $40-80 \mathrm{~K}$ thermal-shield piping should be adequate for a 20 -cryomodule system. In both cases, the cryomodule-string length (i.e., 20 units) would be about 1/10 of that in the ILC, but the pressure drop per unit length would be on the order of 10 times larger. Finally, if ones accounts for cryogenic loss uncertainties, overcapacity factor and other uses for the cryogens, then two 2.5-MW plants (state of the art) could serve the entire machine.

Another important component in the cryomodule is the cavity power coupler. The TTF3-style coupler that has been used at DESY and will be used in XFEL is designed for $\sim 2-\mathrm{kW}$ average power transmission. At the $20-\mathrm{kW}$ level required for the soft $\mathrm{x}$-ray FEL linac, the inner conductor temperature would rise over $500{ }^{\circ} \mathrm{C}$. However, the Cornell ERL group has modified this coupler design including air-cooling the inner conductor and has demonstrated that it can handle over $50 \mathrm{~kW} \mathrm{CW}$. Thus, such a design could be used in this soft x-ray FEL application, with the only change perhaps being to increase the copper-plating thickness of the "warm" inner conductor section. 


\subsubsection{LLRF and HLRF}

To operate the cavities, one challenge will be the high external Q $\left(\mathrm{Q}_{\text {ext }}\right)$. For matched power flow, $Q_{\text {ext }}$ needs to be $1.7 \times 10^{7}$, which is over five time larger than that for ILC but at least five times smaller than that for the Cornell ERL. At $1.7 \times 10^{7}$, the cavity bandwidth is about $75 \mathrm{~Hz}$, so the cavities will have to be precisely tuned and will be sensitive to microphonics (typically less than $10 \mathrm{~Hz}$ rms). However, the RF overhead to compensate for any detuning should not have to be too large (maybe 20\%). Because of this and the CW operation, the LLRF controls and feedback systems should be fairly straightforward to implement. That is, the fields in the cavities powered by each RF source (see below) would be monitored with pickup probes, digitized and summed vectorially. The result would be used as input to a feedback algorithm that would maintain a constant average gradient and phase by controlling the drive to the RF source.

To power $\sim 160$ cavities (e.g., assuming seven cell cavities operating at $20 \mathrm{MV} / \mathrm{m}$ ), there are several options. One would be to use one $\sim 20-\mathrm{kW}$ source per cavity, either an IOT (CPI and Thales builds $20-\mathrm{kW}$ versions with $\sim 60 \%$ efficiency and $23-\mathrm{dB}$ gain) or a klystron (CPI builds a $10-\mathrm{kW}$ version with $50-\mathrm{dB}$ gain and $33 \%$ efficiency at $1.2 \times 10^{-6}$ perveance; at a lower perveance, the efficiency could probably be increased to 50\%). At these power levels, the IOT is probably more cost-effective [51]. However, for a CW electron linac, such fine local control may not be needed. Having one source that would drive an eight-cavity cryomodule, for example, would still allow 20 energy "knobs" for the linac. In this case, one could use a 160-kW klystron to allow adequate overhead at $20 \mathrm{MV} / \mathrm{m}$. CPI makes a 100-kW, 51\%-efficient, 56-dB gain, 1.5-GHz tube [52] that perhaps could modified to produce $200 \mathrm{~kW}$ at $1.3 \mathrm{GHz}$. Also, e2V makes a $1.3-\mathrm{GHz}, 160-\mathrm{kW}(120 \mathrm{~kW}$ with $0.5-\mathrm{dB} / \mathrm{dB}$ incremental gain) klystron with a $\sim 50 \%$ efficiency that the Cornell ERL test injector uses [53].

\subsubsection{Pre-Construction R\&D Plan}

The main design goals during the pre-construction phase would be to finalize the cavitycoupler-HOM geometries, cryomodule layout, and cryogenic system, and to choose the power source and feed system (i.e., number of cavities per source). The most computer-intensive part of this program would be the HOM studies to verify that there are no high $(\mathrm{R} / \mathrm{Q})^{*} \mathrm{Q}_{\text {ext }}$ modes and that the beamline absorbers are effective at dissipating the HOM power at $80 \mathrm{~K}$. Other simulation studies would be done to optimize the cavity shape, verify that multipacting is benign, and finalize the cavity-power and HOM-coupler designs. With about a year's effort, these studies could be completed to a such a degree that cavity-prototype construction could begin. This work would benefit from the several-year effort at Cornell on its ERL design and from similar studies that are underway for ILC at FNAL and at JLAB. After the first year, the design work would continue in support of the prototype studies.

After the cavity design is finalized, a few would be built and tested "vertically" to verify gradient and Q performance, and then they would be fully "dressed" (i.e., with helium vessel, couplers, tuners, absorbers) and high-power tested in a single-cavity cryomodule. This module would also be operated with beam (perhaps at FNAL) to check that there are no obvious high-Q modes. Verifying the absence of these modes with strings of cavities would have to wait until the construction phase. Building and testing three cavities with beam and at power could take advantage of existing appropriate cryogenic plant, and many XFEL/ILC-like parts can be used 
and acquired from existing vendors and laboratories. Completing this within a two-year period would be challenging, although some of the work could start the first year when the design is still being finalized.

\subsection{Laser Systems}

Conventional lasers will play a critical role in next-generation soft x-ray FELs in four key areas: photo-injector drive lasers, beam manipulation and seeding, experimental end-station lasers, and synchronization of all of these lasers across a large-scale facility.

\subsubsection{Injector drive lasers}

The initial creation of a low-emittance bunch of photoelectrons from the cathode will define the maximum electron-beam brightness and ultimate performance of the FEL. Optimizing this process will require significant improvements in the state of the art in cathode and laser design. The cathode material and QE will determine the required laser parameters. High-brightness gun technology for existing or planned short-wavelength FELs use a copper or cesium telluride photocathode and require a UV laser pulse (Section 2.1.2). At the LCLS, 15-20 $\mu \mathrm{J}$ of spatially and temporally shaped $253-\mathrm{nm}$ light is required to produce $250 \mathrm{pC}$ of bunch charge from a copper cathode. Factoring in transport, controls, and diagnostics losses increases the required UV energy output of the laser to the $200-300 \mu \mathrm{J}$ level. The UV is created by harmonically converting 760 -nm light with $\sim 10 \%$ efficiency, therefore $2-3 \mathrm{~mJ}$ of energy is required from the laser system. At the $120-\mathrm{Hz}$ repetition rate, this is a fairly modest average power but scaling to $\mathrm{MHz}$ rates would require $\mathrm{kW}$ average power levels, which are well beyond the current state of the art. This approach would place the majority of the development effort on increasing the average power of the drive laser.

A better approach would use next-generation photocathode materials (Section 2.1) that require much less energy and potentially different wavelengths. This is a significant materials science effort, the outcome of which will determine what laser technology is appropriate. We propose a study of the various laser technologies that would be appropriate for each of the cathode materials under consideration. This study would take into account the expected average power requirement for $100 \mathrm{kHz}$-to-1 $\mathrm{MHz}$ operation, spatial and temporal pulse-shaping requirements, and wavelength conversion to the required wavelength for each of the cathode materials being considered. The goal of this R\&D is to be ready to begin laser development as soon as a cathode material is determined.

\subsubsection{Beam manipulation and seeding}

Beyond the generation of the electron bunch, lasers will be required at the undulator end of the machine for FEL-optimization techniques ranging from bunch manipulation (e.g., ESASE, attosecond pulse production) to seeding (e.g., EEHG and other approaches). In the case of beam manipulation the $\lambda^{2}$ scaling of the ponderomotive interaction implies that much less pulse energy is needed for longer wavelength light. In the case of seeding, a conventional laser will be harmonically converted to the required seed wavelength. Similar interaction scaling with wavelength once again drives the preferred laser to longer wavelength. In either case, the expectation is that up to $\mathrm{mJ}$ level pulses of $\sim 2 \mu \mathrm{m}$ light will be needed to seed at wavelengths of 
order tens of nanometers, which avoids multiple stages of harmonic cascade FELs. Average powers will therefore be well beyond the current state of the art. Because the wavelengths are long and the required pulse widths are short $(<50 \mathrm{fs})$ theses pulses will consist of relatively few electric field oscillations and will in most cases need to be carrier envelope phase (CEP) stabilized.

This is an active area of laser research around the world. Many techniques are currently being explored (and funded by DOE) for generating ultrashort mid-IR pulses. These include: parametric processes, relatively new laser materials (transition-metal-doped chalcogenides such as $\mathrm{Cr}: \mathrm{ZnSe}, \mathrm{Cr}: \mathrm{ZnS}$ ), and thulium- or holmium-doped fiber lasers. In most of these cases, the technology is in the early stages of development and there are significant hurdles to be overcome. Furthermore, some of the technologies proposed here such as EEHG, if successful, will significantly relax the laser-power requirements, thus changing the appropriate laser technology. Section 2.2 discusses seeding techniques and $R \& D$ required.

We propose a study of the ongoing research in mid-IR laser technology in close coordination with the beam-manipulation and seeding studies being proposed in Section 2.2. The outcome of this R\&D would allow laser development to begin as soon as seeding and beam manipulation studies have determined the configuration of the FEL.

\subsubsection{Synchronization}

One of the most compelling reasons for developing next-generation soft x-ray FELs is the ability to study matter on atomic, spatial, and temporal scales. Working on time scales of femtoseconds or less will require significant improvement in the ability to synchronize experimental lasers and equipment to the arrival of the x-rays. The spatial scale of the machines and the distances over which synchronization signals must be transmitted compounds this problem. To stabilize timing in an FEL to the 1-fs level, distances in the x-ray optical path will have to be known and/or controlled to $\sim 300$-nm precision over many hours. Over $\sim 100$-m distances, free-space optical interferometers can be used to measure this long-term precision if their lasers are stable in wavelength to one part per billion. This is about ten times better than most commercial interferometers, although lasers with long-term stabilization to $10^{-11}$ are commercially produced. Typical commercial interferometers are designed only for short-term measurements, sacrificing long-term stability. If a special interferometer must be developed, techniques currently used in commercial interferometers may need to be upgraded, such as evacuating and temperaturestabilizing the reference optical paths. Interferometer optical beamlines could be integrated into the FEL design to closely match the x-ray path. Distance data from the free-space interferometers could be used to rapidly control phase delays in the laser timing system described above, "closing the loop" of timing in the facility. We propose this work be performed under project or other R\&D proposal funding. 


\section{Cost Estimate}

All funding required for performing critical pre-construction R\&D identified in this white paper is listed below, including costs requested through other proposals. Activities are listed in priority order. Labor estimates are based on a fully burdened FTE rate of $\$ 250 \mathrm{k} /$ year (i.e., an average between LBNL and SLAC rates). Procurements are burdened at 10\%, and costs are escalated at $4 \% /$ year. $35 \%$ contingency is included in the totals.

Table 3.1. Summary of R\&D Costs

\begin{tabular}{|c|c|c|c|c|c|}
\hline Activity & FY09 & FY10 & FY11 & FY12 & Total \\
\hline \multicolumn{6}{|l|}{ Injector } \\
\hline FTE & 2.70 & 7.22 & 6.00 & 5.05 & \\
\hline Materials $(\$ M)$ & 0.50 & 1.86 & 2.30 & 0.73 & \\
\hline Total including contingency $(\$ M)$ & 1.65 & 5.40 & 5.88 & 3.13 & 16.06 \\
\hline \multicolumn{6}{|l|}{ FEL design } \\
\hline FTE & 1.95 & 4.67 & 3.20 & 3.20 & \\
\hline Materials $(\$ M)$ & 0.22 & 0.23 & 0.05 & 0.05 & \\
\hline Total including contingency (\$M) & 0.98 & 1.99 & 1.25 & 1.30 & 5.53 \\
\hline \multicolumn{6}{|l|}{ Insertion devices } \\
\hline FTE & 0.50 & 2.00 & 2.25 & 1.25 & \\
\hline Materials (\$M) & 0.10 & 0.34 & 0.29 & 0.19 & \\
\hline Total including contingency $(\$ M)$ & 0.32 & 1.23 & 1.29 & 0.79 & 3.62 \\
\hline \multicolumn{6}{|l|}{ Linac systems } \\
\hline FTE & 0.60 & 6.00 & 4.00 & 4.00 & \\
\hline Materials $(\$ M)$ & 0.35 & 0.60 & 0.60 & 0.25 & \\
\hline Total including contingency $(\$ M)$ & 0.72 & 0.03 & 2.42 & 1.94 & 8.11 \\
\hline \multicolumn{6}{|l|}{ Laser systems } \\
\hline FTE & 0.25 & 0.50 & 0.50 & 0.50 & \\
\hline Materials $(\$ M)$ & 0.02 & 0.02 & 0.02 & 0.02 & \\
\hline Total including contingency $(\$ \mathrm{M})$ & 1.11 & 0.21 & 0.21 & 0.22 & 0.75 \\
\hline
\end{tabular}

\begin{tabular}{|r|r|r|r|r|r|}
\hline Grand total & 3.78 & 11.86 & 11.05 & 7.38 & \\
\hline Cumulative & 3.78 & 15.64 & 26.69 & 34.07 & \\
\hline
\end{tabular}




\section{Core Competencies of institutions}

\subsection{LBNL}

Born as an accelerator laboratory, LBNL has maintained a leading role in accelerator science and technology for seven decades. Today, it applies its wide-ranging expertise to extend the scientific reach of technologies for the production, acceleration, diagnostics, and control of chargedparticle and x-ray beams. Advances in theory, computer modeling, hardware design, and experimental R\&D aim at achieving fundamental understanding beyond the current state of the art that leads to new concepts and systems for advanced x-ray light source facilities with optimized performance, as well as for other applications.

Based on core competencies in and backed up by extensive experimental and theoretical resources for accelerator and x-ray beamline design found in LBNL's Accelerator and Fusion Research (AFRD), Engineering, Advanced Light Source (ALS), Physics, Nuclear Science, Materials Science, and Chemical Science Divisions, the Laboratory's expertise covers a wide range:

- Ion and electron sources.

- Photocathodes.

- Accelerator theory, design, and simulation for ring, RF and induction-linac-based facilities and for laser-plasma-wakefield accelerators.

- High-resolution multi-physics modeling and code development.

- Charged-particle and photon-beam instrumentation and diagnostics.

- Photon production and manipulation in FELs.

- X-ray optics and beamlines.

- High-power lasers and laser-optical systems, including precision timing and synchronization.

- RF structure design, LLRF, and high-power systems.

- Beam feedback and controls.

- Superconducting magnets.

- Mechanical engineering including conventional magnet technologies, undulators, and vacuum systems.

\subsubsection{Research facilities}

Research facilities applicable to this expertise include shielded enclosures for accelerator component and systems testing; high-power RF and HV test stands; research accelerators for beam tests with electrons, protons, and ions; laser laboratories; laser-plasma acceleration laboratories; RF and microwave laboratories; $\mathrm{x}$-ray optics laboratories; and access to operating user facilities and beamlines (ALS, 88-inch cyclotron). The advanced photo-injector experiment 
(APEX) and the LOASIS laboratory are two noteworthy examples of resources available to the R\&D proposed here:

The APEX laboratories consist of an RF photo-injector laboratory and two cathode-development and test laboratories. They are dedicated to the study of new high-brightness, high-repetition-rate photo-injector concepts. Based on normal-conducting RF technology in the VHF band (187 $\mathrm{MHz}$ ) and capable of running in continuous wave mode, the photo-injector is under construction at LBNL. The photo-injector is specially designed to target two main goals: serving as the main part of an R\&D facility for the study and generation at high repetition rates of electron beams with quality compatible with FEL applications and as a versatile photocathode test facility. The cathode laboratories are dedicated development of very high efficiency $(>10 \%)$ photocathodes with properties commensurate with high-gradient photo-guns. This should have tremendous effect on operation at very high $(\mathrm{MHz})$ repetition rates with modest laser sources. Such a facility will represent a formidable tool for investigating beam dynamics and cathode physics and for student training.

The LOASIS laboratory has been performing pioneering research on laser-plasma accelerators for over a decade. Existing equipment include a 100-TW, 30-fs, Ti:sapphire (0.8- $\mu \mathrm{m})$ laser and associated diagnostics; a laser-plasma accelerator that has demonstrated production of $1-\mathrm{GeV}$ electron bunches within a 3-cm-long plasma; and the THUNDER undulator (2.2-cm period, 10 sections, 0.5-m each). Acquisition of a 1-PW, 40-J, 40-fs laser system will occur over the next few years. It is planned to inject the high-current, short-pulse electron beams of high quality produced in the LOASIS Laboratory into an FEL undulator for experiments in lasing. Seeding techniques using laser high-harmonic generation (HHG) will be explored, including production of ultrashort pulses with a few-cycle seed laser.

\subsubsection{R\&D support}

In support of the R\&D proposed here, LBNL can direct much of its existing accelerator physics and engineering expertise and resources toward the proposed program to enhance efficiencies and expedite results.

LBNL has already invested LDRD funds in the APEX effort aimed at an integrated design of accelerating structure, cathode, and laser systems for a high-repetition rate electron photo-gun, of the type identified as critical R\&D for this program. A VHF accelerating cavity is currently under construction, and a power supply is about to be purchased. The integrated photo-gun system will be housed in the Beam Test Facility (BTF), an existing shielded enclosure located at the ALS, with adjacent utilities and infrastructure suitable to support this R\&D development of a high-repetition-rate and high-brightness electron source. A laser system currently under development in an STTR project will be housed above the gun to drive photocathodes that are being developed in a separate test laboratory nearby. The cathode development program takes advantage of the surface and materials sciences expertise at the ALS, the Molecular Foundry, and elsewhere at LBNL.

Space is available within the BTF for building a beamline, additional acceleration components, and diagnostics equipment to characterize the electron beam. The cathode insertion system and 
VHF cavity will accommodate rapid cathode replacement, allowing tests of a variety of photocathode materials in real accelerator environment. The VHF cavity is specifically designed to provide an excellent vacuum for enhanced lifetime of cathodes. We propose to build on these existing investments to synergistically assemble and test the proposed electron source delivering bunches with bunch current up to a nanocoulomb at $\mathrm{MHz}$ repetition rate and with normalized emittance $\leq 1 \mathrm{~mm} \bullet \mathrm{mrad}$. This allows efficiencies and costs reductions compared to pursuing a new program.

In support of undulator development for future light source facilities, LBNL has expertise and infrastructure for superconducting magnet development relevant to the R\&D required for shortperiod and narrow-gap devices identified as cost drivers for a future FEL facility. LBNL has a significant history of undulator development, and a strong group that has designed and produced insertion devices for the ALS and other facilities.

In FEL design, LBNL has made important contributions to the development of concepts for optical manipulations of beams. Previously, the Laboratory has applied optical manipulation of beams using lasers to produce ultrafast x-ray pulses at the ALS in a "laser slicing" technique, now implemented worldwide. More recently, other optical techniques for ESASE being planned for implementation at the LCLS have been developed. ESASE and production of attosecond xray pulses in FELs are examples of leading work performed at LBNL. Techniques for producing powerful incoherent and coherent radiation in a spectral range from terahertz to x-ray with pulse lengths from tenths of picoseconds to tenths of attoseconds are on the horizon.

LBNL's development of diagnostics and instrumentation allow accurate determination of beam properties required for synchronization of accelerator and laser systems to the femtosecond regime. Not only has the Laboratory's research on particle storage rings helped to keep the ALS at the world forefront of synchrotron light sources, but timing and synchronization systems are being designed and built by LBNL for applications at the LCLS and the FERMI@Elettra FEL facilities. Using stabilized optical fibers and propagating RF signals as modulations of an IR fiber-laser carrier wave, better than 20-fs stability has been demonstrated over a 2-km signal path in real-world conditions at the LCLS.

UV and soft X-ray beamline and optics design expertise is widely available at LBNL. The ALS, one of the world's brightest sources of ultraviolet and soft x-ray beams, houses experience in beamline design for multiple applications, including design of the SXR beamline for LCLS. The Center for X-Ray Optics (CXRO) is a facility dedicated to advancing the science and technology of short-wave optical systems, including extreme ultraviolet and soft x-ray radiation. The Ultrafast X-Ray Science Laboratory (UXSL) bridges the gap between development of ultrafast $\mathrm{X}$-ray and extreme ultraviolet sources and their application to problems at the frontiers of the chemical, materials, and biological sciences. The Ultrafast Materials Sciences Program develops sources and techniques important to the emerging capabilities in ultrafast $\mathrm{x}$-ray science.

\subsection{SLAC}

The SLAC National Accelerator Laboratory is operated by Stanford University and is funded by the U.S. Department of Energy. SLAC is a multipurpose laboratory for astrophysics, photon 
science, accelerator and particle physics research. Six scientists have been awarded the Nobel Prize for work carried out at the laboratory. SLAC has a long history of research and development of novel concepts for accelerators including the 2-mile long SLAC linac, the SPEAR storage ring, the Stanford Linear Collider, and, most recently, the Linac Coherent Light Source, the world's first X-ray free electron laser.

SLAC has core competencies and extensive infrastructure to support accelerator research, beamline design, and ultrafast science within the Accelerator Research Division (ARD) and in the Linac Coherent Light Source (LCLS), Stanford Synchrotron Radiation Lightsource (SSRL), Photon Science (PS), and Engineering and Technical Services (ETS) Directorates. The accelerator-research and beamline-design expertise covers a wide range from fundamental accelerator theory to facility design, construction, and operation. This expertise includes design and simulations for

- Particle sources.

- Storage rings.

- Bunch compressors.

- Normal-conducting and superconducting RF linacs.

- Dielectric and plasma wakefield accelerators.

- Photon production, x-ray optics and beamlines.

- FELs.

Particular strengths are in characterizing coherent effects, electromagnetic modeling and code development using massively parallel processing and in RF source and structure design. Hardware, instrumentation, and beam-control expertise includes

- Electron and positron sources including state-of-the-art RF guns.

- High-power RF sources and structures.

- LLRF and timing.

- Charged particle and photon beam instrumentation and diagnostics.

- Beam feedback and controls.

- High-power lasers and laser/optical systems.

- Mechanical engineering including conventional magnet technologies, alignment, and vacuum systems.

\subsubsection{Research facilities}

At present, SLAC operates two synchrotron radiation user facilities SSRL (based on SPEAR3, a third-generation storage ring) and the LCLS. SSRL supports about 2000 users annually. It operates with an electron energy of $3 \mathrm{GeV}$ and has a brightness exceeding $10^{19} \mathrm{ph} / \mathrm{s} / \mathrm{mm}^{2} / \mathrm{mrad}^{2} / 0.1 \% \mathrm{BW}$ in the $1-10 \mathrm{keV}$ spectral range at $500 \mathrm{~mA}$. The LCLS is the world's first X-ray FEL. Commissioning of the LCLS was started in the spring of 2009. The FEL has already lased at $1.5 \AA$ and has exceeded many of its design goals, including achieving 
saturation with less than half the full complement of undulators and achieving better than expected shot-to-shot photon stability. The experience with the LCLS design, construction, and operation will be critical for future FEL projects.

SLAC also has numerous test facilities for accelerator research, including the Next Linear Collider Test Accelerator (NLCTA), the Accelerator Structure Test Area (ASTA), FACET, which is being constructed, and the proposed Injector Test Facility (ITF). ASTA is a test facility that can be used for high-power testing of RF components, RF gun development, and photocathode development; the proposed Cathode Test Facility would be constructed as part of ASTA.

The NLCTA at SLAC is a $~ 300-\mathrm{MeV} \mathrm{X}$-band linac with an RF photo-injector electron source capable of producing high brightness electron beams. A range of near- and mid-IR laser beams that are precisely timed to the electron beam are available for conducting laser-electron interaction research. Successful attosecond bunch-train formation, diagnosis, and acceleration experiments have already been carried out at this facility. The NLCTA can also be used for component testing or configured for a variety of beam physics experiments.

SLAC is presently seeking internal LDRD funding to construct a proof-of-principle demonstration of echo-enhanced harmonic generation (EEHG) at the $7^{\text {th }}$ harmonic of a $1.57-\mu \mathrm{m}-$ wavelength seed laser, yielding microbunch structure at $224 \mathrm{~nm}$, relying on conventional UV optics for diagnosis. Beam quality and energy are expected to support generation of the $16^{\text {th }}$ harmonic, and a follow on experiment using the same undulators and chicanes at the NLCTA will diagnose the microbunching (at $98 \mathrm{~nm}$ ) using an in-vacuum spectrometer.

Both FACET and ITF will use the first $2 \mathrm{~km}$ of the linac tunnel. FACET has an experimental area at the end of $2^{\text {nd }} \mathrm{km}$ of the linac with high-energy-density electron and positron beams of over $20 \mathrm{GeV}$ with peak currents in excess of $20 \mathrm{kA}$ that can be focused to transverse spot sizes of $10 \mu \mathrm{m}$. The Injector Test Facility (ITF) is an upgrade-in-planning of the SLAC main linac injector at Sector 0 to support generation and acceleration of LCLS-quality beams. This facility is seeking funding under a separate proposal. Once commissioned, beams of sufficient density ( $1 \mathrm{~mm}-\mathrm{mr}, 1 \mathrm{kA}$ ) and energy (up to $24 \mathrm{GeV}$ ) would be available to fully test seeding and beam manipulation concepts in the relevant parameter range.

In addition to the onsite test facilities, SLAC participates in many strong collaborations. The LCLS project is a collaboration led by SLAC among Department of Energy laboratories including Argonne, Brookhaven, Los Alamos, and Lawrence Livermore national laboratories as well as the University of California, Los Angeles. SLAC also leads many of the R\&D efforts of the International Linear Collider Global Design Effort, a collaboration of over 20 institutions from around the world designing a superconducting linear collider. SLAC is working closely with Fermilab in the construction of a new large superconducting RF test facility located at Fermilab. SLAC collaborates closely with KEK on the ATF/ATF2 test facility, which produces some of the world's lowest-emittance beams with a goal of focusing the beams down to $35 \mathrm{~nm}$ at $1.3 \mathrm{GeV}$. Finally, SLAC is home to the PULSE Institute for ultrafast energy science, a highly collaborative BES research center. PULSE scientists have broad expertise in ultrafast laser and $\mathrm{x}$-ray techniques, with applications to AMO, biological, chemical, condensed matter and 
materials science.

\subsubsection{R\&D support}

In support of the R\&D described in this proposal, SLAC can direct much of its accelerator physics and engineering expertise and resources toward the proposed program. SLAC can utilize the accelerator research test facilities for the development and demonstration of the critical beam manipulation techniques such as enhanced SASE (ESASE) and EEHG harmonic generation. For example, SLAC plans use the NLC Test Accelerator to demonstrate the EEHG concept, a novel seeding technique that was proposed by the SLAC Accelerator Beam Physics group. In addition, there is an outstanding proposal to use the LCLS to demonstrate the ESASE concept, a technique of generating short, temporally coherent, radiation pulses.

The SLAC Accelerator Beam Physics group can contribute to the detailed FEL design with a combination of analytic studies, simulations, and parameter specification using tools that have been benchmarked against the LCLS FEL. Using expertise from the International Linear Collider R\&D program, the LCLS design effort, the Beam Physics and Advanced Computation groups SLAC can also contribute to the beam transport design including the particle beam optics and simulation and the wakefield/impedance calculations using tools that

SLAC has a strong program in superconducting linac R\&D and is a major participant in the design of the superconducting linac of the International Linear Collider and the DOE-funded SCRF R\&D program. SLAC can apply this expertise to contribute to the superconducting linac design including the parameter specification and systems integration, the cavity design and RF power source design. In addition, the suite of massively parallel electromagnetic simulations that have been developed at SLAC can be applied to perform detailed optimization of the cryomodule, superconducting cavity and coupler designs. These codes have been recently compared against measurements of the JLab 12-GeV Upgrade cavities and then used to reoptimize the cavity design.

Using expertise from the International Linear Collider R\&D program in collaboration with Lawrence Livermore National Laboratory, SLAC has developed fast kicker pulsers that exceed the required specifications for the FEL beam switchyard. These systems can be demonstrated at the ATF test facility at KEK, a SLAC collaborator, where the low emittance $1.3 \mathrm{GeV}$ beam can be used to verify the performance and stability with high resolution.

Finally, using expertise from the PULSE Institute, SSRL, and the LCLS, SLAC can contribute to the design and specification of the laser systems and diagnostics required for beam generation and beam manipulation and to the VUV and soft x-ray beamline optics design. 


\title{
5 Other Institutions
}

Several other institutions have expertise and infrastructure in test stands and facilities that may be applied to this R\&D program. We list here those institutions that we anticipate involving in these studies:

\author{
Argonne National Laboratory \\ Brookhaven National Laboratory \\ Cornell University \\ Fermi National Accelerator Laboratory \\ Lawrence Livermore National Laboratory \\ Massachusetts Institute of Technology \\ Oak Ridge National Laboratory \\ Thomas Jefferson National Accelerator Facility \\ University of California, Los Angeles \\ University of Wisconsin- Madison
}




\section{References}

\section{Chapter 1}

1. Basic Energy Sciences Advisory Committee, U.S. Department of Energy, "New Science for a Secure and Sustainable Energy Future," December 2008. Available online at http://www.sc.doe.gov/bes/reports/list.html.

2. Basic Energy Sciences Advisory Committee, U.S. Department of Energy, "Next-Generation Photon Sources for Grand Challenges in Science and Energy," May 2009. Available online at http://www.sc.doe.gov/bes/reports/list.html.

3. Basic Energy Sciences Advisory Committee, U.S. Department of Energy, "Directing Matter and Energy: Five Challenges for Science and the Imagination,” December 20, 2007. Available online at http://www.sc.doe.gov/bes/reports/list.html.

4. Argonne National Laboratory, Brookhaven National Laboratory, Lawrence Berkeley National Laboratory, and SLAC National Accelerator Laboratory, "Science and Technology of Future Light Sources," December 2008. Available online at http://www.als.lbl.gov/als/publications/genpubs.html.

5. Lawrence Berkeley National Laboratory. "Toward Control of Matter: Energy Science Needs for a New Class of X-Ray Light Sources," September 2008. Available online at https://hpcrd.lbl.gov/sxls/home.html.

\section{Chapter 2}

6. See for example reference 5 above.

7. B.E. Carlsten, Nuclear Inst. and Methods in Physics Research A 285, 313 (1989).

8. I. Bazarov, C. Sinclair, Phys. Rev. ST Accel. Beams 8034202 (2005).

9 C.Pellegrini, private communications.

10. C. Hernandez-Garcia, et al., Proc. of 26th International Free Electron Laser Conference, Trieste, Italy (2004) p. 558.

11. B. M. Dunham, et al., Proc. of 2007 IEEE Part. Accel. Conf., Albuquerque, NM, USA (2007) p. 1224.

12. See for example:

Å. J. Teichert, et al., Proc. of the 27th International Free Electron Laser Conference, Stanford, CA, USA (2005) 534.;

A. M. M. Todd, et al., Proc. of 2005 IEEE Part. Accel. Conf.,Knoxville, TN, USA (2005) 2292.

13. D. Janssen and V. Volkov, Proc. of 2004 European Particle Accelerator Conference, Lucerne, Switzerland (2004) p. 330. 
14. D. H. Dowell, et al., Proc. of the 2007 Electron Laser Conference, Novosibirsk, Russia (2007) p. 276.

15. J . W. Staples, S. P. Virostek and S. M. Lidia, Proc. of the 2004 European Particle Accelerator Conference, Lucerne, Switzerland, July (2004) 473.

16. S.S. Kurennoy, et al., Nuclear Inst. and Methods in Physics Research A 528, 392 (2004).

17. R. A. Rimmer, Proceedings of the 2005 Particle Accelerator Conference, Knoxville, TN USA (2005) 3049.

18. J. Staples, F. Sannibale, S. Virostek, CBP Tech Note 366, Oct. 2006 or: K. Baptiste, et al, Nuclear Inst. And Methods in Physics Research A 599, 9 (2009).

19. X. Chang, et al., Proc. Of the 2007 IEEE Part. Accel. Conf., Albuquerque, NM, USA (2007) p. 2547.

20. A. H. Sommer, Photoemissive Materials. 1968: John Wiley.

21. I. V. Bazarov, I.V., B.M. Dunham, and C.K. Sinclair, Physical Review Letters, 2009. 102 (10): p. 104801.

22. C. Ghosh, Physical Review B, 1980. 22(4): p. 1972.

23. D. H. Dowell, S.Z. Bethel, and K.D. Friddell, Nucl. Inst. Meth., 1995. 356(2-3): p. 167.

24. K. Bane, P. Emma, PAC2005 Proceedings, (2005).

25. M. Borland, Advanced Photon Source LS-287, 2000.

26. M. Dohlus, T. Limberg, FEL2004 Proceedings, 18 (2004).

27. J. Qiang et. al., Phys. Rev. ST-AB 9, 044204 (2006).

28. M. Venturini, R. Warnock, A. Zholents, Phys. Rev. Special Topics - Acc. and Beam, 10, 054403 (2007).

29. K. Bane et. al., Phys. Rev. ST-AB 12, 030704 (2009).

30. P. Emma et. al., PAC2009 Proceedings, 2009.

31. Z. Huang et. al., Phys. Rev. ST-AB 7, 074401 (2004).

32. Linac Coherent Light Source Conceptual Design Report, SLAC-R-593, 2002.

33. Fermi@Elettra conceptual design report http://www.elettra.trieste.it/FERMI, Elettra (2007).

34. G. Stupakov, Phys. Rev. Lett. 102, 074801 (2009).

35. D. Xiang, G. Stupakov, Phys. Rev. ST-AB 12, 030702 (2009).

36. L.-H. Yu et. al., Science 289, 932 (2000).

37. A. Zholents, K. Holdack, Free Electron Laser Conference, FEL06, Berlin, Germany, (2006).

38. S. Reiche, Nucl. Instrum. Methods Phys. Res. A 429, 243 (1999).

39. W. Fawley, Report LBNL-49625, 2002.

40. W. Ackermann et al., Nature photonics 1, 336 (2007). 
41. P. Emma for the LCLS commissioning team, PAC2009 Proceedings, 2009.

42. A.H. Zewail, J. Phys. Chem. A, 104, 5660(2000).

43. A. Zholents,Laser Physics, Vol. 15, No. 6, p.855, 2005.

44. J. Stohr, H. Siegmann, Magnetism: From Fundamentals to Nanoscale Dynamics (Springer, Berlin, 2006).

45. K.-J. Kim, Nucl. Instrum. Methods A 445, 329 (2000).

46. Y. Ding, Z. Huang, Phys. Rev. ST-AB 11, 030702 (2008).

47. M. Liepe and J. Knobloch, Nuclear Instruments and Methods in Physics Research A 557 (2006) 354-369.

48. M. Liepe, "Conceptual layout of the cavity string of the Cornell ERL main linac cryomodule,"

49. G. Ciovati, P. Kneisel†, J. Brawley, R. Bundy, I. Campisi, K. Davis, K. Macha, D. Machie, J. Mammosser, S. Morgan, R. Sundelin, L. Turlington, K. Wilson, Jefferson Lab; M. Doleans, S.H. Kim, D. Mangra, ORNL-SNS; D. Barni, C. Pagani, P. Pierini, INFN Milano; K. Matsumoto, R. Mitchell, D. Schrage, LANL; R. Parodi, INFN Genova; J. Sekutowicz, DESY; P. Ylae-Oijala, "Superconducting prototype cavities for the Spallation Neutron Source (SNS) project,"

50. Stuart Henderson, "Spallation Neutron Source progress, challenges and upgrade options," Proceedings of EPAC08, Genoa, Italy.

51. A. Zolfghari, P. MacGibbon, B. North, "Comparison of klystron and inductive output tubes (IOT) vacuum-electron devices for RF amplifier service in free-electron laser," Proceedings of EPAC 2004, Lucerne, Switzerland.

52. S. Lenci, H. Bohlen, B. Stockwell, E. Wright, and Al Mizuhara, "Recent progress in CW klystrons at CPI," Proceedings of EPAC 2002, Paris, France.

53. S. Belomestnykh\#, J. Dobbins, R. Kaplan, M. Liepe, P. Quigley, J. Reilly, C. Strohman, V. Veshcherevich, "Commissioning of the Cornell ERL injector RF systems," Presented at the 2008 European Particle Accelerator Conference, Genoa, Italy. 


\section{Acknowledgements}

This document was coordinated by, and includes written contributions from, the team listed at the front of the document. In addition, we acknowledge helpful comments from:

Ricki Campisi (ORNL), Georg Hoffstaetter (Cornell University), Henry Kapteyn (KMLabs), Tom Peterson (FNAL) 


\title{
8 Appendix. Resumes of Principal Participants
}

Resumes are in alphabetical order.

\author{
Curriculum Vitae \\ CHRIS ADOLPHSEN
}

$\underline{\text { POSITION }}$

Physicist at the Stanford Linear Accelerator Center since 1990

Address: $\quad$ MS 66

2575 Sand Hill Road

Menlo Park, CA 94025

Phone: $\quad$ 650-926-3560

Email: $\quad$ star@slac.stanford.edu

Web Page: http://www-user.slac.stanford.edu/star/

\section{FELLOWSHIP}

American Physical Society, Division of Physics of Beams in 2003 "for original contributions to the beam physics and microwave properties of high frequency, high gradient linear accelerators."

\section{EDUCATION}

University of Chicago, Chicago, Illinois

Dates: $\quad$ October 1978 - June 1985

Degrees: $\quad$ Ph.D. (December 1985)

Specialty: $\quad$ Experimental High Energy Physics

University of Rochester, Rochester, New York

Dates: $\quad$ September 1974 - May 1978

Degrees: $\quad$ BS (cum laude)

Major: Physics

Honor: William Eastwood Scholarship 


\section{Eric R. Colby \\ Advanced Accelerator Research Department \\ Stanford Linear Accelerator Center, 2575 Sand Hill Road, Menlo Park, CA 94025 \\ Phone: (650) 926-3709 Email: ecolby@slac.stanford.edu}

\section{EDUCATION}

Ph. D., Physics, University of California Los Angeles 1997. Dissertation title: "Design, Construction, and Testing of a Radiofrequency Electron Photo-injector for the Next Generation Linear Collider".

\section{PROFESSIONAL HISTORY}

Head, Advanced Accelerator Research Department, SLAC, 2008-present. Deputy Head, Advanced Accelerator Research Department 2006-present

Staff Physicist, Stanford Linear Accelerator Center, 2005-present.

Experiment E-163 Spokesman 2001-present

W. K. H. Panofsky Fellow, Stanford Linear Accelerator Center, 2000-2005.

Research Associate, Stanford Linear Accelerator Center, 1998-2000.

Research Associate, Fermi National Accelerator Laboratory 1997-1998.

\section{SELECTED PROFESSIONAL ACCOMPLISHMENTS}

RF Photo-injector Modeling \& Construction. Developed the prototype and first production RF gun for the DESY TESLA SASE FEL. Extensive code development to model electron emission, space charge effects, and realistic accelerator properties. Installed and commissioned 4 RF guns and either managed or played a key role in the design and construction of 3 RF gun facilities at ANL, FNAL, and SLAC.

Millimeter-Wave Power Generation. Provided the first comprehensive models of sheet beam klystron performance for efficient generation of $95 \mathrm{GHz}$ power at megawatt levels. Simulated, fabricated, and measured RF cavities, antennae, diffraction masks and other millimeter-wave components.

Laser-DrivenElectron Bunching and Acceleration. Spokesman for SLAC experiment E-163, Laser Acceleration of Electrons at the NLCTA, an experiment to optically bunch and efficiently accelerate electrons with light, leading to attosecond electron bunches. The experiment has demonstrated the production of attosecond bunches, and subsequent acceleration.

\section{SELECTED PUBLICATIONS}

1. C. M. S. Sears et al, "Phase stable net acceleration of electrons from a two-stage optical accelerator", $P R S T-A B$, 11, 101301, (2008).

2. C. M.S. Sears et al, "Production and characterization of attosecond electron bunch trains" PRSTAB, 11, 061301, (2008).

3. E. Colby, P. Musumeci, "Summary Report of Working Group 7: Electromagnetic-Structure based Accelerators", AAC06, AIP Conf. Proc. 877, pp. 183, (2006).

4. T. Plettner, R. Byer, E. Colby, B. Cowan, C. M. S. Sears, J. E. Spencer, and R. H. Siemann, "Proof-of-principle experiment for laser-driven acceleration of relativistic electrons in a semiinfinite vacuum", $P R S T$ - AB, 8, 121301 (2005).

5. T. Plettner, R. Byer, E. Colby, B. Cowan, C. Sears, J., Spencer, R. Siemann, "Visible-Light Acceleration of Relativistic Electrons in a Semi-Infinite Vacuum”, Phys. Rev. Lett., 95, 134801 (2005).

6. C. M. Sears, E. Colby, B. Cowan, R. Siemann, J. Spencer, R. Byer, T. Plettner, "High Harmonic Inverse Free Electron Laser interaction at 800 nm", Phys. Rev. Lett., 95, 194801 (2005).

7. L. Schachter, E. Colby, R. Siemann, "Saturation of bunch-wave interaction in an active medium", Phys. Rev. Lett .87, 134802, (2001).

8. J. Andruszkow, et al, "First Observation of Self-Amplified Spontaneous Emission in a Free- Electron Laser at 109 nm Wavelength", Phys. Rev. Lett., 85, pp.3825, (2000).

9. J. Rosenzweig, E. Colby, "Charge and Wavelength scaling of RF Photo-injector designs", Aip Conf. Proc. 335, pp. 724 (1995).

10. E. Colby, D. Dowell, et al, "An Injector Test Facility for the LCLS”, SLAC-TN-07-005, (2007). 


\section{John N. Corlett}

Staff Scientist/Physicist

Accelerator and Fusion Research Division

Lawrence Berkeley National Laboratory

1 Cyclotron Road, MS 71R0259, Berkeley, CA 94720

(510) 486-5228; (510) 486-7981 (fax); JNCorlett@1bl.gov

\section{Education}

1980 - 1983: B.Sc. Physics, University of Liverpool, England

\section{Research Interests}

Collective effects in charged particle beams. Beam impedance measurements and computation. Design of radiofrequency, microwave, millimeter-wave, and optical structures, devices, and systems for the acceleration, control, and diagnostics of charged-particle beams for next-generation particle accelerators, including broadband feedback systems. Storage ring design, linac-based synchrotron radiation facilities, FEL facility design.

\section{Professional Experience}

Staff Scientist, Lawrence Berkeley National Laboratory

$1993-$

Deputy Division Director, AFRD, (2008-); Program Head, Center for Beam Physics (2004-); Technical Director, LUX Project (2002-4); Group Leader, Beam Electrodynamics Group (1993-2003); Research in: Damping Rings design; RF systems for ionization cooling; collective effects, RF, and feedback systems for PEP-II.

Scientist, Lawrence Berkeley National Laboratory

$1991-1993$

Research in: collective effects and feedback systems for ALS, impedance measurements.

Daresbury Laboratory, Warrington, United Kingdom

$1986-1991$

Accelerator Physicist, Synchrotron Radiation Source (SRS) Accelerator Physics Group

EEV Co. Ltd., Lincoln, United Kingdom

$1983-1986$

Microwave physicist/engineer.

\section{Recent Professional Services}

Member Fermi Research Alliance Visiting Committee; Chair of the Fermilab Accelerator Advisory Committee; Member of the PEP-II B-factory Machine Advisory Committee; Member of the LCLS Facility Advisory Committee; Member-at-large of the American Physical Society Division of Physics of Beams; Member of the ILCAmericas Global Design Effort (GDE) regional team.

\section{Selected Publications}

J. N. Corlett \& R. O. Hettel, "Performance Requirements and Metrics for Future X-Ray Sources", Proc. PAC09 (Vancouver, BC, 4-8 May 2009), paper TU5RFP038

"A CW normal-conductive RF gun for free electron laser and energy recovery linac applications", K. Baptiste, J. Corlett, S. Kwiatkowski, et al, NIM A, Vol. 599, Issue 1, 1 February 2009, pp 9-14

“FERMI@Elettra - A Seeded Harmonic Cascade FEL for EUV and Soft X-rays”, (with C. Bocchetta, et al.), Proc. $27^{\text {th }}$ International FEL Conference FEL2005, Stanford, CA, August 2005, FROA003.

"LUX - A design study for a Linac/Laser-based Ultrafast X-ray source", J. N. Corlett, W. A. Barletta et al, Proc. Fourth Generation X-Ray Sources and Optics II, Denver, August 2004, SPIE Proc. Vol. 5534.

"805 MHz and $201 \mathrm{MHz}$ RF cavity development for MUCOOL”, D. Li, J. Corlett, A. Ladran et al, J. Phys. G: Nucl. Part. Phys. 29 (2003) 1683-1687

"Techniques for Synchronization of X-ray Pulses to the Pump Laser in a Ultrafast X-ray Facility", J.N. Corlett, L. Doolittle, R. Schoenlein, J. Staples, R. Wilcox, and A. Zholents, Proc. PAC2003, Portland, Oregon, May 2003.

"Extremely low vertical-emittance beam in accelerator-test facility at KEK", (with K. Kubo et al), Phys. Rev. Lett. 88, 194801 (2002).

"RF Deflecting Cavity Design for the Berkeley Ultrafast X-ray Source", D. Li, J. Corlett, Proc. $8^{\text {th }}$ European Particle Accelerator Conference, Paris, June 3-7, 2002. 


\section{David H. Dowell CURRICULUMVITA}

e-mail: dowell@slac.stanford.edu

\section{EDUCATION}

- Doctor of Philosophy in Experimental Nuclear Physics (1981)

University of Illinois, Champaign-Urbana, Illinois

- Master of Science in Physics (1974)

University of Illinois, Champaign-Urbana, Illinois

- Bachelor of Engineering Physics (1972)

University of Illinois, Champaign-Urbana, Illinois

\section{EMPLOYMENT HISTORY}

- October 2001-May 2008 Staff Physicist

Linac Coherent Light Source

Stanford Linear Accelerator Center

Menlo Park, CA

- July 1987 - August 2001 Associate Technical Fellow

Principal Investigator

Free Electron Laser Program

Boeing Physical Sciences Research Center

Seattle, WA

- $\quad$ Oct. 1985 - June 1987

Associate Physicist

Physics Department

Brookhaven National Laboratory

Upton, NY

- $\quad$ Oct. 1983 - Oct. 1985

Assistant Physicist

Physics Department

Brookhaven National Laboratory

Upton, NY

- Nov. 1980 - Sept. 1983 Postdoctoral Research Associate

Nuclear Physics Laboratory

University of Washington

Seattle, WA

\section{PROFESSIONAL INTERESTS}

My primary interests are the generation, preservation and use of high-charge, high-brightness electron beams for driving free electron lasers (FELs). I have performed FEL experiments at visible wavelengths using a five-meter wiggler with both concentric and ring-resonator cavity configurations and operated a photocathode RF injector a very high-average current. Since 2001 I've been at Stanford Linear Accelerator Center working on the Linac Coherent Light Source (LCLS) which is now the world's first xray free electron laser, where I managed the design, construction and commissioning of the electron injector. 


\section{JOHN N. GALAYDA \\ SLAC National Accelerator Laboratory \\ 2575 Sand Hill Road, MS-103 \\ Menlo Park, CA 94025-7015}

\section{EDUCATION}

1977, Ph.D., Physics, Rutgers University

1970, BA (magna cum laude), Physics, Lehigh University

\section{Professional/Academic}

2008-

2007-2008

2005-

2001-

1999-2001

1990-1999

1987-1990
Director, LCLS Strategic Projects Division, SLAC

Associate Laboratory Director, Linac Coherent Light Source, SLAC

Professor (Research), SLAC National Accelerator Laboratory Particle Physics/Astrophysics Faculty - Professor, Photon Science Faculty

Director, Linac Coherent Light Source Project, Stanford Linear Accelerator Center

Deputy Associate Laboratory Director, Advanced Photon Source, Argonne National Laboratory

Director of the Accelerator Systems Division, Advanced Photon Source, Argonne National Laboratory

Associate Chairman for Accelerators, National Synchrotron Light Source, Brookhaven National Laboratory

\section{AWARDS \& HONORS}

Elected a Fellow of The American Physical Society, 1996

R\&D 100 Award for global feedback orbit control, 1989

Elected to Phi Beta Kappa, 1970

\section{PRINCIPAL PUBLICATIONS}

1. S. V. Milton, E. Gluskin, S. G. Biedron, R. J. Dejus, P. K. Den Hartog, J. N. Galayda, K. J. Kim,J. W. Lewellen, E. R. Moog, V. Sajaev, N. S. Sereno, G. Travish, N. A. Vinokurov, N. D. Arnold, C.

Benson, W. Berg, J. A. Biggs, M. Borland, J. A. Carwardine, Y. C. Chae, G. Decker, B. N. Deriy, M. J. Erdmann, H. Friedsam, C. Gold, A. E. Grelick, M. W. Hahne, K. C. Harkay, Z. Huang, E. S. Lessner, R. M. Lill, A. H. Lumpkin, O. A. Makarov, G. M. Markovich, D. Meyer, A. Nassiri, J. R. Noonan, S. J. Pasky, G. Pile, T. L. Smith, R. Soliday, B. J. Tieman, E. M. Trakhtenberg, G. F.Trento, I. B. Vasserman, D. R. Walters, X. J. Wang, G. Wiemerslage, S. Xu, B. X. Yang, "Observation of Self-Amplified Spontaneous Emission and Exponential Growth at $530 \mathrm{~nm}$," Phys. Rev. Lett., 85, 5, July, pp. 988-991, (2000).

2. J. Galayda, "The Advanced Photon Source," Proceedings of the 1995 Particle Accelerator Conference and International Conference on High Energy Accelerators, Dallas, TX, May 1-5, 1995, Vol. 1, 4-8 (1996)

3. M. C. Teich, T. Tanabe, T. C. Marshall, J. Galayda, "Statistical Properties of Wiggler and Bending Magnet Radiation from the Brookhaven Vacuum-Ultraviolet Electron Storage Ring," Physical Review Letters, 65, (27), 3393-3396 (December 1990).

4. L. H. Yu, R. Biscardi, J. Bittner, E. Bozoki, J. Galayda, J. Krinsky, S. Nawrocky, O. Singh, G. Vignola, "Real Time Closed Orbit Correction System," Nucl.Instrum.Meth. A284:268-285, 1989 


\section{Zhirong Huang \\ SLAC, Stanford University \\ 2575 Sand Hill Road, Menlo Park, CA 94025 \\ Phone: (650) 9263947 \\ Email: zrh@slac.stanford.edu}

\section{EDUCATION:}

B.S. in Physics, California Institute of Technology, 1992

Ph.D. in Physics, Stanford University, 1998

\section{PROFESSIONAL HISTORY AND EXPERIENCE:}

Physicist, SLAC National Accelerator Laboratory, 10/2002-present

Research in high-brightness beams and x-ray free electron lasers. Studied accelerator physics issues associated with the generation and preservation of high-brightness beams. Analyzed a detrimental microbunching instability in the LCLS and designed a laser heater to suppress the instability. Explored various short electron and x-ray pulse generation schemes. Worked on novel gain and coherence enhancement methods for x-ray FELs. Participated in the LCLS linac and FEL commissioning.

Physicist, Argonne National laboratory, 6/1998-9/2002

Research in high-brightness beams and x-ray free electron lasers. Performed comprehensive analysis on selfamplified spontaneous emission process. Developed a three-dimensional analysis of nonlinear harmonic generation in high-gain FELs. Carried out data analysis and simulations for the LEUTL FEL that first demonstrated SASE saturation. Formulated a theory of coherent synchrotron radiation microbunching in bunch compressors.

\section{SELECTED AWARDS:}

1999 Outstanding Doctoral Thesis Research in Beam Physics Award

\section{SELECTED PUBLICATIONS:}

1. K. Bane et al., "Measurements and Modeling of Coherent Synchrotron Radiation and its Impact on the LCLS Electron Beam," Phys. Rev. ST Accel. Beams 12, 030704 (2009).

2. Y. Ding and Z. Huang, "Statistical Analysis of Crossed Undulator for Polarization Control in a SASE FEL," Phys. Rev. ST Accel. Beams 11, 030702 (2008).

3. Z. Huang and K.-J. Kim, "Review of X-ray FEL Theory," Phys. Rev. ST Accel. Beams 10, 034801 (2007).

4. Z. Huang and R. Ruth, "Fully Coherent X-ray Pulses from a Regenerative Amplifier Free Electron Laser," Phys. Rev. Lett. 96, 144801 (2006).

5. Z. Huang and G. Stupakov, "Free Electron Lasers with Slowly Varying Beam and Undulator Parameters," Phys. Rev. ST Accel. Beams 8, 040702 (2005).

6. Z. Huang et al., "Suppression of Microbunching Instability in the Linac Coherent Light Source," Phys. Rev. ST Accel. Beams 7, 074401 (2004).

7. Z. Huang and K.-J. Kim, "Formulas for CSR Microbunching in a Bunch Compressor Chicane," Phys. Rev. ST Accel. Beams 5, 074401 (2002).

8. S.V. Milton et al., "Measured Exponential Gain and Saturation of a SASE Free-Electron Laser," Science 292, 2037 (2001).

9. Z. Huang and K.-J. Kim, "Three-Dimensional Analysis of Harmonic Generation in High-Gain Free-Electron Lasers," Phys. Rev. E 62, 7295 (2000).

10. Z. Huang and R.D. Ruth, "Laser-Electron Storage Ring," Phys. Rev. Lett. 80, 976 (1998). 
Howard A. Padmore

Group Leader

Advanced Light Source Division

Lawrence Berkeley National Laboratory

1 Cyclotron Road, MS 2R0400, Berkeley, CA 94720

510-486-5787 HAPadmore@lbl.gov

\section{Research Interests}

Materials science using x-ray synchrotron techniques. Current interests center around investigation of magnetic surfaces using photoemission microscopy, elucidation of stress in thin films using micro-focussed $x$-ray diffraction, ultra-fast dynamics in solids using fsec laser - x-ray pump probe techniques, use of laser alignment in $\mathrm{x}$-ray scattering from molecules in solution, and solid state physics of photocathodes

\section{Education}

Ph.D., Leicester University, UK (1983), Soft X-ray Emission Spectroscopy of the Heavy Rare Earths", B.Sc. (physics), Leicester University (1977).

\section{Recent Professional Experience}

Group Leader, Lawrence Berkeley National Laboratory

4/93-

Division Deputy for Experimental Systems Group (ESG) at the Advanced Light Source (ALS). ALS is a 3rd generation synchrotron $\mathrm{x}$-ray source of high brightness VUV and soft $\mathrm{x}$-ray radiation and has additional capabilities in the hard $\mathrm{x}$-ray domain.

Member of the editorial board of the (UK) Institute of Physics Journal of Measurement Science and Technology, J. Phys. E 91-94

Member of the international Scientific Advisory Committees of:

Sincrotrone Trieste, Italy

99-05

Pohang Light Source, Korea

1999-

Synchrotron Radiation Research Center, Taiwan

00-03

CAMD (2004 - 2005 ): Diamond Light Source, UK

2004

Stanford Synchrotron Radiation Laboratory (SSRL)

$2005-$

Member of the editorial board of the Journal of Synchrotron Radiation

1999

\section{Selected Research Highlights 2000 - 2009:}

A. Scholl et al., "Observation of anti-ferromagnetic domains in epitaxial LaFeO3 by means of x-ray magnetic linear dichroism spectromicroscop,y” Science 287: 1014-1016.

A. M. Lindenberg et al., "Time resolved $x$-ray diffraction from coherent phonons during a laser induced phase transition," PRL 84, 111.

F. Nolting., et al., "Direct observation of exchange alignment of ferromagnetic by antiferromagnetic domains, " Nature 405, 767 (2000).

R. Spolenak, et al., “Local Plasticity of Al Thin Films as Revealed by X-Ray Microdiffraction,” PRL 90, 096102-14.

W. A. Caldwell, et al., "Shear at Twin Domain Boundaries in $\left.\mathrm{YBa}_{2}\right] \mathrm{Cu}_{3} \mathrm{O}_{7-x}$," PRL 92(21): 216105-4.

P. Goudeau, et al., "Mesoscale x-ray diffraction measurement of stress relaxation associated with buckling in compressed thin films, " APL 83(1):51-53.

S. B. Choe et al., "Vortex core-driven magnetization dynamics" Science 304(5669): 420-422.

Bartelt et al, "Element specific spin and orbital dynamics of FeGd multilayers," APL 90,162504. 


\section{Soren O. Prestemon}

1 Cyclotron Road, Mailstop 46R0161

Berkeley, Ca, 94720

\section{EXPERIENCE:}

6/02-present Research Scientist/Engineer, Lawrence Berkeley National Laboratory, Eng. Division

- Advanced Light Source and Accelerator and Fusion R\&D Divisions

6/01-6/02 Engineering Intern, Lawrence Berkeley National Laboratory, Eng. Division

10/98-3/01 Engineering Analyst, Magnet Science \& Technology, National High Magnetic Field Laboratory, Tallahassee, Florida

01/94-3/01 Research Assistant, Mechanical Engineering, Florida A\&M University-Florida State University College of Engineering, Tallahassee, Florida

1/88-9/92 Research Assistant, Max-Planck Institute / Laboratoire des Champs Magnetiques Intense (High Field Magnet Laboratory), Grenoble, France

\section{EDUCATION:}

Florida State University $\quad$ Mechanical Engineering $\quad$ Ph.D $\quad$ (2001)

Institut National Polytechnique, Grenoble, France Mechanical Engineering $\quad$ M.S. (1993)

Institut Joseph Fourier, University of Grenoble, France Mathematics $\quad \sim$ B.A. (1992)

\section{RESEARCH INTERESTS}

- Magnet design for high-energy accelerators and for synchrotron radiation sources

- Permanent, resistive, and superconducting magnet system design

- Project management for the engineering of complex experiments

\section{SERVICE AND AWARDS}

- Member, Program Committee, Particle Accelerator Conference 2009

- Member, Program Committee, Applied Superconductivity Conference 2006, 2008

- Member, Program Committee, Magnet Technology Conference 2007

- $\quad$ Editor, CHATS-AS Workshop, LBNL, 2006

- Co-Instructor:

- Fund. of Accelerator Physics and Technology, USPAS, Jan., 2006; June 2007

- Superconducting Magnets for Accelerators, USPAS, June 2007, June 2009

- Magnetic Systems: Insertion Device Design, USPAS, January, 2008

- Chair, External Oversight Committee, Series Connected Hybrid, NHMFL, 2005-2007

- $\quad$ Klaus Halbach Award, Advanced Light Source, LBNL 2005

- $\quad$ Outstanding Performance Award, LBNL, 2003

- $\quad$ Best Paper award, ICMC 1999

\section{PUBLICATIONS (Selected):}

1. S. Prestemon et al., "High Performance Short-Period Undulators Using High Temperature

2. Superconductor Tapes", proceedings, PAC 2009, Vancouver, Canada

3. Soren Prestemon, Steve Marks, Ross Schlueter, "New Developments in Light Source Magnet Design", Invited paper, PAC 2007, Albuquerque, NM

4. D. Dietderich et al., "Fabrication of a Short-Period Nb3Sn Superconducting Undulator", IEEE Trans. Appl. Supercond., VOL. 17, NO. 2, JUNE 2007, pp 1243-1246

5. A. Godeke et al., "Limits of NbTi and Nb3Sn, and development of W\&R Bi-2212 high field accelerator magnets,” IEEE Trans. Appl. Supercond.,vol. 17, no. 2, p. 1149, 2007.

6. S. Prestemon, R. Schlueter, S. Marks, D. Dietderich, "Superconducting Undulators with Variable Polarization and Enhanced Spectral Range", IEEE Transactions on Applied Superconductivity, June 2006.

7. R. Schlueter, S. Marks, S. Prestemon, D. Dietderich, "Superconducting Undulator Research at LBNL", Synchrotron Radiation News, Jan./Feb. 2004, Vol. 17, No. 1.

8. S. Prestemon, D. Dietderich, S. Marks, R. Schlueter, "NbTi and $\mathrm{Nb}_{3} \mathrm{Sn}$ superconducting undulator designs”, presented at SRI 2003, San Francisco, Aug. 2003. Synchrotron Radiation Instrumentation, AIP, vol. 705, p 294, 2004. 


\section{FERNANDO SANNIBALE}

Staff Scientist

Advanced Light Source Accelerator Physics Group - LBNL

Berkeley, CA 94530, USA

Phone +1 5104865924 - Email: fsannibale@lbl.gov

\section{Education:}

10/84-02/91: 'Laurea' in Physics with maximum grade at the First University of Rome "La Sapienza". Referees Prof. G.V. Pallottino and Dr. A. Vignati.

Professional Experience:

09/04-present, Staff Scientist (career position), ALS Accelerator Physics Group, Lawrence Berkeley National Laboratory, CA, USA.

05/01-08/04, Electronic Engineer 4, ALS Accelerator Physics Group, Lawrence Berkeley National Laboratory, CA, USA.

06/91-04/01, Accelerator Researcher, Accelerator Division, Laboratori Nazionali di Frascati, Istituto Nazionale di Fisica Nucleare, Frascati, Italy

\section{Present research activity:}

High brightness electron source, beam instrumentation and diagnostics and coherent synchrotron radiation in accelerators.

\section{Ten Selected Publications:}

1. F. Sannibale, et al., A Model Describing Stable Coherent Synchrotron Radiation in Storage Rings, Physics Review Letters, Volume 93, 94801 (2004).

2. K. Baptiste, J. Corlett, S. Kwiatkowski, S. Lidia, J. Qiang, F. Sannibale, K. Sonnad, J. Staples, S. Virostek, R. Wells, A CW normal-conductive RF gun for free electron laser and energy recovery linac applications. Nuclear Inst. \& Methods in Physics Research A 599, 9 (2009).

3. F.Sannibale, G. Stupakov, M. Zolotorev, D. Filippetto, L. Jagerhofer, Absolute bunch length measurements by incoherent radiation fluctuation analysis, Phys. Review Special Topics Accelerators and Beams 12, 032801 (2009).

4. J. M. Byrd, Z. Hao, M. C. Martin, D. S. Robin, F. Sannibale, R.W. Schoenlein, A. A. Zholents, M. S. Zolotorev, Laser Seeding of the Storage-Ring Microbunching Instability for High-Power Coherent Terahertz Radiation, Phys. Rev. Letters 97, 074802 (2006).

5. F. Sannibale, A. Marcelli and P. Innocenzi, IKNO, a user facility for coherent terahertz and UV synchrotron radiation. Journal of Synch. Radiation 15, 655 (2008).

6. M.S. Zolotorev, E. D. Commins, F.Sannibale, Proposal for a Quantum-degenerate Electron Source, Physical Review Letters 98, 184801 (2007).

7. J. M. Byrd, W.P. Leemans, A. Loftsdottir, B. Marcelis, M. C. Martin, W. R. McKinney, F .Sannibale, T. Scarvie, C. Steier. Observation of Broadband Self-Amplified Spontaneous Coherent Terahertz Synchrotron Radiation in a Storage Ring. Physical Review Letters, Volume 89, 224801 (2002).

8. J. M. Byrd, Z. Hao, M. C. Martin, D. S. Robin, F. Sannibale, R.W. Schoenlein, A. A. Zholents, M. S. Zolotorev. Tailored terahertz pulses from a laser-modulated electron beam, Physical Review Letters, Volume 96, 164801 (2006).

9. G.Mazzitelli, A. Ghigo, F.Sannibale, P. Valente, G. Vignola, Commissioning of the DA ФNE Beam Test Facility, Nuclear Instr. \& Methods in Phys. Research A 515 (2003) 524.

10. G.Mazzitelli, F.Sannibale, F. Cervelli, T. Lomtadze, M. Serio, G. Vignola, Single Bremsstrahlung luminosity measurements at DA $\Phi N E$, Nuclear Instruments \& Methods in Physics Research A 486 (2002) 568. 


\section{CURRICULUM VITAE - BIOGRAPHICAL SKETCH}

Name:

Ross D. Schlueter

RDSchlueter@1bl.gov
Position Title:

Deputy Director, Engineering Div.; Dept. Head, Mechanical Eng. Lawrence Berkeley National Laboratory, Berkeley, CA 94720

Education/Training:

Institution and Location

Stanford University, Palo Alto, CA

Stanford University, Palo Alto, CA

Georgia Inst. of Tech., Atlanta, GA
Degree

Ph.D.

M.S.

B.S.
Year Conferred, Field of Study

1984, Magnetohydrodynamics

1980, Thermal Sciences

1979, Mechanical Engineering

Positions:

1990-present (Senior) Staff Scientist, Lawrence Berkeley National Laboratory, Berkeley, CA

1987-1990 R\&D Engineer, Lawrence Livermore National Laboratory, Livermore, CA

1983-1987 R\&D Engineer, Weyerhaeuser Company, Tacoma, WA

1979-1983 Research Assistant, Stanford University, Palo Alto, CA

1977-1978 Plant Engineer, General Motors, Fisher Body Fleetwood, Detroit, MI

1975-1975 Baker, Bäckerei Neumaier, Rieden bei Füssen, Bayern

Affiliations and Service:

American Physical Society

American Society of Mechanical Engineers

Institute of Electrical and Electronics Engineers

Reviewer for Editorial Boards of Nucl. Mag. Instr., Rev. Sci. Instr., and DOE SBIR Invited Instructor- graduate courses in Magnet Technology at: UC Santa Cruz (2008), Yale (2002), Beijing (1998), Stanford (1998), Berkeley (1997), Duke (1995), Hsinchu (1993), Florida State (1991)

\section{Honors:}

2002 LBL Outstanding Performance Award: ALS hysteresis-free Chicane Performance 2001 LBL Halbach Award for Synchrotron Instrumentation: ALS Superbend Project 1995 LBL Outstanding Performance Award:ALS Undulator Performance 1993 LBL Outstanding Performance Award: ALS Insertion Device Theory and Design 1980 Stanford University Charles Hawley Memorial Fellowship 1979 Rotary Club of Atlanta Award: Most Outstanding Senior at Ga. Inst. of Tech. 1979 Pi Tau Sigma Award: Most Outstanding Senior in Mech. Eng. at Ga. Inst. of Tech. 1977 General Motors Corporation Fellowship

Patents and inventions:

Invention Discl. Reversed-biased, magnetically-enhanced electron microscope magnet, Jan 2007 U.S. Patent \#7535229 Rotating NMR/MRI magnet, May 2009

U.S. Patent \#6015476 Plasma reactor magnet w. indep. controllable current elements, Jan 2000 Invention Disclosure Compact, tunable, high field multipole, May 1997 Invention Disclosure Tunable pure permanent magnet harmonics corrector ring, January 1996 U.S. Patent \#5099175 Tunability enhanced electromagnetic wiggler March 1992 


\section{Dr. Carl B. Schroeder}

University of California at Berkeley, Ph.D., Physics, 1999; M.A., Physics, 1995.

University of Maryland at College Park, B.S., Mathematics, 1994; B.S., Physics, 1994.

Dr. Carl Schroeder is a Staff Scientist in the Accelerator\& Fusion Research Division of the Lawrence Berkeley National Laboratory. His research interests include novel radiation sources, free-electron lasers, plasma-based accelerators, and intense laser-plasma interactions.

Dr. Schroeder joined the Lawrence Berkeley National Laboratory in 2002. His recent research has concentrated in the areas of laser-driven plasma wave excitation, short-pulse laser-plasma in- stabilities, and radiation generation from beam-plasma interactions. Dr. Schroeder received the Lawrence Berkeley National Laboratory Outstanding Performance Award in 2005 and 2007. From 2000 to 2001, Dr. Schroeder was a postdoctoral fellow at the University of California, Los Angeles, where his research focused on the development of a high-gain x-ray free-electron laser. His graduate work at the University of California, Berkeley concerned the study of laser-plasma and beam-plasma interactions with the goal of developing plasma-based accelerators. In 1995, Dr. Schroeder was a Visiting Scholar in the Plasma Fusion Center at the Massachusetts Institute of Technology. In 1994 he was awarded a Department of Energy Fusion Science Fellowship, and in 1993 he won the University of Maryland Dorfman Prize for Outstanding Undergraduate Research.

Dr. Schroeder has published over 45 papers in refereed scientific journals.

Selected Journal Publications:

Dr. Carl Dr. Carl Schroeder Schroeder

E. Esarey, C. B. Schroeder, andW. P. Leemans, "Physics of laser-driven plasma-based electron accelerators," Rev. Mod. Phys. (2009).

C. B. Schroeder, E. Esarey, E. Cormier-Michel, W. P. Leemans, "High-harmonic generation in cavitated plasmas," Phys. Plasmas 15, 056704 (2008).

W. P. Leemans, B. Nagler, A. J. Gonsalves, Cs. T'oth, K. Nakamura, C. G. R. Geddes, E. Esarey, C. B. Schroeder, S. M. Hooker, "GeV electron beams from a centimetre-scale accelerator," Nature Phys. 2, 296 (2006).

C. B. Schroeder, E. Esarey, B. A. Shadwick, and W. P. Leemans, "Trapping, dark current, and wavebreaking in nonlinear plasma waves," Phys. Plasmas 13, 033103 (2006).

C. B. Schroeder, E. Esarey, and B. A. Shadwick, "Warm wavebreaking of nonlinear plasma waves with arbitrary phase velocities,” Phys. Rev. E 72, 055401(R) (2005).

C. B. Schroeder, E. Esarey, and W. P. Leemans, "Electron-Beam Conditioning by Thomson Scattering," Phys. Rev. Lett. 93, 194801 (2004).

C. G. R. Geddes, Cs. T'oth, J. van Tilborg, E. Esarey, C. B. Schroeder, D. Bruhwiler, C. Nieter, J. Cary, and W. P. Leemans, "High quality electron beams from a plasma channel guided laser wakefield accelerator," Nature 431, 538 (30 September 2004).

C. B. Schroeder, E. Esarey, J. van Tilborg, andW. P. Leemans, "Theory of coherent transition radiation generated at a plasma-vacuum interface," Phys. Rev. E 69, 016501 (2004).

C. B. Schroeder, E. Esarey, B. A. Shadwick, and W. P. Leemans, "Raman forward scattering of chirped laser pulses," Phys. Plasmas 10, 285 (2003).

C. B. Schroeder, D. H. Whittum, and J. S. Wurtele, "Multimode Analysis of the Hollow Plasma Channel Wakefield Accelerator,” Phys. Rev. Lett. 30, 1177 (1999). 
SLAC National Accelerator Laboratory

William (Bill) White

M/S 102, 2575 Sand Hill Rd.

Menlo Park, CA 94025

Office: 650-926-2290, Cell: 408-313-0134

wewhite@slac.stanford.edu

\section{Professional Background:}

4/05 - present Laser Group Leader: SLAC National Accelerator Laboratory, Stanford University, Menlo Park, CA. Responsible for developing and managing the Laser Group in support of LCLS, LUSI, PULSE, as well as other SLAC laser projects.

1/07 - present Consultant: Technical consultant on high power, ultrafast laser systems , manufacturing techniques, and applications.

12/03 - 4/05 Director of Engineering: Coherent Inc., Santa Clara, CA. Responsible for product development and engineering for the Advanced Systems Business Unit. Also responsible for support of manufacturing, sales and service as well as strategic planning for the business unit.

4/99 - 12/03 Vice President: Positive Light Incorporated, Los Gatos, CA. Involved in all aspects of corporate management while directly responsible for Engineering/R\&D Department. Member of the team which negotiated the acquisition of Positive Light by Coherent Inc.

7/89 - 4/99 Senior Scientist: Lawrence Livermore National Laboratory, Livermore, CA. Facility Manager for Physics Dept. Janus Laser Facility: Responsible for development, maintenance, operation and scheduling of four high intensity laser systems for plasma physics research. Also managed mobile remote sensing laboratory. Job responsibilities included: Funding proposals, budgeting, supervision of scientific and technical staff.

6/97-9/00 Member, Board of Directors: Positive Research Incorporated, San Diego, CA

2/96 - 4/03 Member and Secretary, Board of Directors: Positive Light Incorporated, Los Gatos, CA.

5/92 - 5/99 Consultant: Positive Light Incorporated, Los Gatos, CA.

6/93 - 6/94 Consultant: Spectra-Physics Lasers Incorporated, Mountain View, CA.

9/88 - 3/89 Consultant: Naval Oceanographic Research and Development Agency, Stennis Space Center, Bay St. Louis, MI.

\section{Education:}

Ph. D. in Physics: Texas A\&M University, College Station, TX, awarded 12/89.

B.S. in Physics: Texas A\&M University, College Station, TX, awarded 06/83. 


\section{Russell Wilcox}

Electronic Engineer

Engineering Division

Lawrence Berkeley National Laboratory

1 Cyclotron Road, MS 71R0259, Berkeley, CA 94720

510-495-2704 RBWilcox@1bl.gov

\section{Education}

University of California, Berkeley, California, 1975 - 1980, B.S., Electrical Engineering, June 1980.

\section{Recent Professional Experience}

Lawrence Berkeley National Laboratory

2003-

Designed and built a high average power femtosecond titanium sapphire laser system for the Advanced Light Source, to provide for ultrashort $\mathrm{x}$-ray pulse generation. This is one of the highest average power, high repetition rate femtosecond lasers in operation, demonstrating designs that have been transferred to industry. Designed and built fiber optic systems for synchronizing lasers and RF in accelerator facilities. Applied for patent on new concept in this field.

Phaethon

00-03

Designed and built systems for writing gratings into fibers using UV lasers, receiving several patents for innovative designs.

Lawrence Livermore National Laboratory

80-00

Designed master oscillator laser systems for inertial confinement fusion (ICF). Early innovations include a patented system for driving electro-optic modulators with precisely programmable high voltage pulse shapes, solving a long-standing problem in oscillator development. Developed a new pulse generation approach based on fiber lasers and waveguide modulators, which allowed greater complexity while improving reliability. Most advanced fusion lasers worldwide now use this approach.

\section{Selected Publications}

“Optical Synchronization Systems for Femtosecond X-ray sources”, (with J. Staples et al.), Proceedings of 2005 Particle Accelerator Conference, Knoxville, Tennessee, p 3958

"Stimulated Brillouin scattering thresholds for square pulses in polarizing fiber", (with R. Fluck et al.), Conference on Lasers and Electro-optics 2000, paper CWK17

“Two-stage, Co-pumped, Yb:silica Fiber Amplifiers", (with D. F. Browning et al.), Conference on Lasers and Electro-optics 1998, paper CWK3

"Development System Performance of the NIF Master Oscillator and Pulse Forming Network”, (with D. Browning et al.), Third Annual International Conference on Solid State Lasers for Application to Inertial Confinement Fusion, 1998 SPIE Proceedings vol. 3492, p. 100

"Fusion Laser Oscillator and Pulse-forming System Using Integrated Optics”, (with D. F. Browning et al.), in Laser Coherence Control: Technology and Applications, 1993, SPIE proceedings vol. 1870, p. 53

“New Approach to Fusion Laser Master Oscillator Design”, Conference on Lasers and Electro-optics 1992, paper CFA7

“Precise Shaping of Kilovolt Pulses with Subnanosecond Resolution”, IEEE Conference Record of the 1986 Seventeenth Power Modulator Symposium, 1986, p. 201 


\section{Alexander A. Zholents}

Senior Scientist

Accelerator and Fusion Research Division

Lawrence Berkeley National Laboratory

1 Cyclotron Road, MS 71R0259, Berkeley, CA 94720

510-486-7533_AAZholents@lbl.gov

\section{Fellowships and Awards}

Fellow of the American Physical Society, 2005.

Lawrence Berkeley National Laboratory outstanding performance award, 1999, 2004.

Klaus Halbach award for innovative instrumentation, 2000.

\section{Education}

Ph.D. in Experimental High-Energy Physics from Budker Institute of Nuclear Physics, Siberian Branch of Russian Academy if Science, Novosibirsk, Russia. 1983.

M.S. in Physics from Physics Department of the Institute of Electrical Engineering, Novosibirsk, Russia. 1973.

\section{Recent Professional Experience}

Senior Scientist, Lawrence Berkeley National Laboratory

$2001-$

Staff Scientist, Lawrence Berkeley National Laboratory

Visiting Scientist, European Center for Nuclear Research, Geneva, Switzerland $91-92$

Leading Scientist, Budker Institute of Nuclear Physics, Novosibirsk, Russia 89-91

Senior Scientist, Budker Institute of Nuclear Physics, Novosibirsk, Russia

Junior Scientist, Budker Institute of Nuclear Physics, Novosibirsk, Russia $\quad$ 78-84

Post Graduate Scientist, Budker Institute of Nuclear Physics, Novosibirsk, Russia 75-78

Researcher, Budker Institute of Nuclear Physics, Novosibirsk, Russia 73-75

\section{Selected Publications}

"Laser Seeding of the Storage-Ring Microbunching Instability for High-Power Coherent Terahertz Radiation" (with J. Byrd et al.), Physical Review Letters, 97, (2006) 74802.

"Tailored terahertz pulses from a laser-modulated electron beam" (with J. Byrd et al.), Physical Review Letters, 96, (2006) 164801.

"Fermi@ Elettra: A Free Electron Laser for EUV and soft X-ray radiation" (with C. Bocchetta et al.), Synchrotron Radiation News 18N6, (2005)30; LBNL 60135.

"Generation of short x-ray pulses using crab cavities at the advanced photon source" (with K. Harkay et al.), Proceedings of 2005 Particle Accelerator Conference, Knoxville, Tennessee, (2005) 668.

“Attosecond X-ray Pulses from Free-Electron Lasers” Laser Physics, 15, No. 6, p.855, 2005.

“Obtaining Attosecond X-ray Pulses Using a Self-Amplified Spontaneous Emission Free Electron Laser” (with G. Penn), Phys. Rev. ST -Acc. And Beams, 8, 050704, 2005.

"Laser Assisted Electron Beam Conditioning for Free Electron Lasers" Phys. Rev. ST Acc. And Beams, 8, 050701, 2005.

"Method of an Enhanced Self-Amplified Spontaneous Emission for X-ray Free Electron Lasers" Phys. Rev. ST Acc. And Beams, 8, 040701, 2005.

"LUX- A recirculating linac-based ultrafast x-ray source" (with J.N. Corlett et al.), 8-th International Conference on Synchrotron Radiation and Instrumentation, AIP. American Institute of Physics Conference Proceedings, no.705, 2004, p.121. USA

"Proposal for Intense Attosecond Radiation from an X-Ray Free-Electron Laser" (with W.M. Fawley), Phys. Rev. Lett., 92, 224801 (2004).

"Optical Stochastic Cooling for RHIC Using Optical Parametric Amplification” (with M. Babzien et al.), Phys. Rev. ST -Acc. And Beams, 7, 012801 (2004).

"Faster, Brighter, Shorter" (with J.N. Corlett et al.), CERN Courier, p.1, October 2003. 